\title{
META-ANALYSIS OF THE GLOBAL DIVERSITY AND SPATIAL PATTERNS OF APHID-ANT MUTUALISTIC RELATIONSHIPS
}

\author{
SidDiQUi, J. A. ${ }^{1,2}-$ LI, J. ${ }^{1,2}-$ ZoU, X. ${ }^{1,2}-$ BODLAH, I. ${ }^{3}-$ HUANG, X. ${ }^{1,2^{*}}$ \\ ${ }^{1}$ State Key Laboratory of Ecological Pest Control for Fujian and Taiwan Crops, College of \\ Plant Protection, Fujian Agriculture and Forestry University, Fuzhou 350002, China \\ ${ }^{2}$ Fujian Provincial Key Laboratory of Insect Ecology, Fujian Agriculture and Forestry \\ University, Fuzhou 350002, China \\ ${ }^{3}$ Laboratory of Biosystematics, Department of Entomology, Pir Mehr Ali Shah Arid Agriculture \\ University, Rawalpindi, Pakistan \\ *Corresponding author \\ e-mail: huangxl@fafu.edu.cn; phone: +86-591-8370-5205
}

(Received $10^{\text {th }}$ Dec 2018; accepted 21 $1^{\text {st }}$ Mar 2019)

\begin{abstract}
Mutualism is a fundamental relationship that can shape species communities through coevolutionary adaptations and also has great ability to change ecosystem functions. Aphids and ants are common insect groups which are distributed widely across the world. The mutualistic relationships between them have gained much attention in history. However, very few studies have been carried out on the diversity of associated aphid and ant species pairs. In this study, we tried to gather available published data about the diversity of species pairs of aphids and ants to find out potential ecological and geographical patterns. The results showed that 284 species of aphids belonging to 83 genera and 9 subfamilies were mutualistically associated with 193 species of ants belonging to 38 genera and 4 subfamilies. In this report we elucidated a total of 972 aphid-ant interactions and 915 ant-aphid interactions (one aphid species may associate with many ant species and vice versa). Among these relationships, a highest number of ant species were associated with the aphid genus Aphis, while a highest number of aphids were associated with the ant genus Formica. Besides, an obvious geographical pattern was indicated that the subtropical region may have a higher diversity of aphid-ant associations. This global analysis of current knowledge about diversity and patterns of aphid-ant mutualistic relationships also indicates that more taxonomic and ecological studies are needed to enrich our understanding of these associations.
\end{abstract}

Keywords: multispecies interaction, symbiosis, myrmecophiles, Hymenoptera, Formicidae

\section{Introduction}

Reciprocally beneficial associations (e.g. mutualism) support and promote biodiversity (Herre et al., 1999; Bascompte and Jordano, 2007; Ito and Gobin, 2009). These interactions are common and conspicuous in terrestrial communities from tropical to temperate regions (Buckley, 1987; Hölldobler and Wilson, 1990; Delabie, 2001; Styrsky and Eubanks, 2007). These associations trigger the dynamics of ecological communities by influencing the environmental variables, species evolution and survival rate (Bronstein, 2009; Hosseini et al., 2017). One classic example of mutualistic relationship are the interactions between aphids and ants (Darwin, 1859; Stadler and Dixon, 2005).

The aphids (Hemiptera: Aphididae) are a group with 24 subfamilies and more than 5000 species all over the world (Favret, 2018). The diversity of aphid species is far higher in the northern hemisphere temperate regions than anywhere else (Dixon et al., 1987; Heie, 1994; Huang et al., 2012). Some aphid species are destructive pests and voracious feeder of various crops, and also vectors of various plants diseases (Mittler, 1958; 
Blackman and Eastop, 1984; Buruchara et al., 2010). The ants (Hymenoptera: Formicidae) are one of the most common insect group on the Earth, which is divided into 21 subfamilies with 497 genera and more than 26700 species (Ward, 2013). Ants usually are very diverse and economically important social insects mostly dominant in the tropical regions (Hölldobler and Wilson, 1990; Philpott and Armbrecht, 2006). Ants fulfill their dietary requirements by performing various roles like decomposers, pollinators, scavengers, predators, and herbivores (Blackman and Eastop, 1984). Ants also feed on honeydew secretions of hemipterans such as aphids, and have a symbiotic association among them (Jahn and Beardsley, 1996; Styrsky and Eubanks, 2007).

Mutualism is a fundamental element in aphid-ant communities and behavior through co-evolutionary adaptation (Herre et al., 1999; Carabalí-Banguero et al., 2013). These relationships can abundantly be found within various aphid-ant groups (Styrsky and Eubanks, 2007) and can shape the species richness, abundance, and distribution. There are two main groups of aphids; myrmecophilous (tended by ants) and non-myrmecophilous (untended by ants) (Novgorodova, 2002, 2005). Myrmecophilous aphids and ants share their habitats and build a strong bond by providing food and security. Ants collect honeydew produced by aphids, and in return they protect them from their predators, parasitoids, and adverse climatic conditions (Hölldobler and Wilson, 1990; Stadler and Dixon, 2005; Novgorodova and Ryabinin, 2017). Moreover, ants provide aphids protection from the sooty mold infestation by sanitation, which propagates in the presences of honeydew accumulation (Way, 1963; Hölldobler and Wilson, 1990; Stadler and Dixon, 2005; Novgorodova and Ryabinin, 2017). For example, the population of associated aphids increased due to these relationships, and they can damage host plants and reduce yield. Honeydew-producing hemipterans tended by ants have a wide range of host plants, including trees, shrubs, vines (Floate and Whitham, 1994; Rico-Gray and Castro, 1996; Styrsky and Eubanks, 2007), fobs and grasses (Moya-Ragoza and Nault, 2000). Besides, the degree of such protection was observed to differ among aphids attending by ants (Addicott, 1978; Katayama and Suzuki, 2003; Novsgorodova and Gavrilyuk, 2012) and also depend on the aggressiveness of ants and honeydew collection approaches, in particular degree of specialty among foragers (Novgorodova and Gavrilyuk, 2012; Novgorodova, 2015). Due to the close aphid-ant interactions, complex networks of mutualistic interactions are formed between these insect groups of multispecies communities. The reason for their successful interaction is that they exist in a wide range of natural habitats (Buckley, 1987; Way and Khoo, 1992). However, ethological and ecological mechanisms of mutualism are still poorly understood.

The majority of previous studies have mainly focused on one or few pairs of aphid-ant species, while the general information of trophobiotic interactions among aphids and ants in most of regions are still limited. Besides, many review papers demonstrated the mutualistic ecology, cost and constraints, positive or negative mutualistic interactions of aphids and ants (Boucher et al., 1982; Buckley, 1987; Bronstein, 1994; Stadler and Dixon, 1998; Hoeksema and Bruna, 2000; Bronstein and Barbosa, 2002; Stadler and Dixon, 2005). However, there is a lack of the knowledge of diversity of aphid-ant species pairs which can provide complete information about their relationships in different ecological zones and can elaborate the patterns of these associations. A reason for this gap may be due to the lack of integration and meta-analysis of dispersed information on aphid-ant interactions in previous literature.

This study will generate an interest in quantifying the importance of aphid-ant interactions for global diversity patterns, distribution of species pairs and will also help 
the examination of the interactions of aphid-ant species across different ecological zones. Furthermore, data on species pairs along with geographical information can be used to study aphid-ant interactions at regional and global scale. Therefore, there is a need to investigate the current state of knowledge about the diversity of aphid-ant species associations. Based on data from published literature, this paper aimed to depict the current state of the taxonomic diversity of aphid-ant species pairs as well as the spatial patterns of their interactions (Tylianakis et al., 2008; Gilman et al., 2010).

\section{Materials and methods}

\section{Data collection of aphid-ant species pairs}

We collected species and distribution data from the available previously published researches (1906 to 2016) on species pairs of mutualistic aphids and ants (Table Al in the Appendix). Literature searching was done by using tools including Web of Science, Google Scholar and Goolge. The data such as names of subfamilies, tribes, genera, and species as well as locations of aphids and associated ants were extracted from published researches. Many articles contained only species names and location, so for getting more details about their higher taxa the databases of Aphid Species File (http://aphid.speciesfile.org) and AntWeb (https://www.antweb.org) were used. These databases provide the complete taxonomic hierarchy of aphids and ants. The validation of species names was also rechecked based on these taxonomic databases. The Aphid Species File contained comprehensive information about aphids from all over the world and provided the taxonomic keys, taxa hierarchy, bibliographic data of aphids from all parts of the world. The AntWeb provides taxonomic keys, taxa hierarchy, specimen records, and natural history information and distribution maps on ants. Up till now, all types of biodiversity data of almost all species has been updated on these databases. The data in these two databases are freely accessible. Other information was fetched from the available published researches in different journals. Distribution localities for available species pairs along with geographical coordinates were compiled and arranged into a database (Microsoft Excel). Species localities were identified from the descriptions of previous literature. The accurate locality data points were collected according to the literature description, google earth and by using the website (www.findlatitudeandlongitude.com).

\section{Geographical patterns of species associations}

Latitudes and longitudes of species pairs were plotted on the outline of the World map with the help of Arc Map 10.2 (Geographical Information System, GIS) software. The ecological zones were selected by using the coordinates of temperate $\left(35-66.5^{\circ}\right)$, subtropical $\left(23.5-35^{\circ}\right)$ and tropical $\left(0-23.5^{\circ}\right)$ zones. The coordinates of temperate, subtropical and tropical regions were separated from primary data set. Separated data was plotted according to the coordinates of temperate, tropical and subtropical regions to find out the diversity of aphid-ant interactions in different regions.

\section{Data analysis}

Data analysis was conducted by using correlation test. For percentage of ant-associated aphids, non-parametric Spearman's test (two-tail) was used while for percentage of aphidassociated ants parametric Pearson test (two-tail) was used. To calculate the percentages 
of genera and their species, the genera and species of each subfamily was divided by the total genera and species in all subfamilies. The fundamental statistical analysis has been applied to find out the diversity of species pairs in different regions of the world. The bar graphs were developed in Microsoft Excel by using extracted data.

\section{Results}

\section{Taxonomic diversity}

\section{Aphid-associated ants}

The percentage of all species numbers failed the normality test, so the nonparametric Spearman's test was used (two-tailed $\mathrm{P}<0.01$ ) for aphid associated ants. The results showed a positive correlation between the number of aphid species in relation with ant species $(\mathrm{t}=0.983, \mathrm{df}=8, \mathrm{P}<0.001)$. Their patterns can be seen in (Table 1). Subfamily Aphidinae with about $59.85 \%$ of total aphid species was found associated with four subfamilies of ants (Table 1). Out of total 83 genera, the genus Aphis of subfamily Aphidinae was chiefly associated with $70.5 \%$ of ant species (Table 1).

Out of nine genera of subfamily Calaphidinae, genus Symydobius were associated with maximum number of ant species from four genera. Among the four genera of subfamily Chaitophorinae, about $81.25 \%$ species of the genus Chaitophorus were associated with 16 genera of ants. Out of 10 genera of the subfamily Eriosomatinae, the genus Pemphigus (only one species) was found supremely associated with 11 genera of ants. Among the eight genera of subfamily Lachninae, about $74.57 \%$ species of ants were interacting with the genus Cinara. In subfamily Drepanosiphinae the genus Drepanaphis having more interactions with ants. Genus Thelaxes received more ants than other genera under the subfamily Thelaxinae. For subfamilies Anoeciinae and Saltusaphidinae, only one genus from each was associated with ants (Table 1). Except subfamilies Thelaxinae and Saltusaphidinae, all genera from the other 7 subfamilies were mainly associated with species of ant genus Formica.

Table 1. The diversity of ant-associated aphids, with the numbers and percentages of aphid genera and species in each subfamily. The information of associated ants for each aphid group are also provided

\begin{tabular}{c|c|c|c|c|c|c|c}
\hline \multicolumn{4}{c|}{ Aphid } & \multicolumn{3}{c}{ Aphid-associated ant } \\
\hline Subfamily name & Genus & Genus \% & Species & Species \% & Subfamily & Genus & Species \\
\hline Anoeciinae & 1 & 1.204 & 1 & 0.352 & 1 & 1 & 2 \\
Aphidinae & 46 & 55.421 & 170 & 59.859 & 4 & 37 & 161 \\
Calaphidinae & 9 & 10.843 & 12 & 4.225 & 2 & 6 & 15 \\
Chaitophorinae & 4 & 4.819 & 29 & 10.211 & 3 & 16 & 53 \\
Drepanosiphinae & 2 & 2.409 & 3 & 1.0563 & 2 & 3 & 6 \\
Eriosomatinae & 10 & 12.048 & 11 & 3.873 & 3 & 11 & 23 \\
Lachninae & 8 & 9.638 & 53 & 18.661 & 3 & 14 & 59 \\
Thelaxinae & 2 & 2.409 & 4 & 1.408 & 3 & 7 & 13 \\
Saltusaphidinae & 1 & 1.204 & 1 & 0.352 & 2 & 2 & 2 \\
Total & 83 & & 284 & & & & \\
\hline
\end{tabular}




\section{Ant-associated aphids}

The data was found normal through the normality test, so the parametric Pearman's test was applied (two-tailed $\mathrm{P}<0.01$ ) for ant-associated aphids. The results showed a positive correlation between the numbers of aphid species and that of ant species $(\mathrm{t}=0.983, \mathrm{df}=3, \mathrm{P}=0.009)$. The data presented graphically in Table 2. Out of four ant subfamilies, the Formicinae had maximum associations (66.84\% of all) with all nine aphid subfamilies. The genus Formica was found highly associated with a maximum number of aphid species. Subfamily Myrmicinae contributes $29.53 \%$ of all associations with aphids, and out of the 16 genera associated with aphids, genus Myrmica tended maximum aphid species. Genus Tapinoma of subfamily Dolichoderinae found higher associations with aphids out of 6 other genera. Only genus Tetraponera of subfamily Pseudomyrmecinae interacted with only one species of ant (Table 2). Genus Aphis was found highly associated with almost all genera of all ant subfamilies.

\section{Geographical diversity}

Geographical diversity of species pairs varies in different geographical zones. Figure 1 showed that the maximum aphid-ant species pairs were in the subtropical region. The ratios of species numbers of aphid to ant in these geographical zones were measured as 1.9:1 in the temperate, 1:2.8 in tropical while nearly equal associations with the ratio 1.1:1 in the subtropical region. In the temperate zone, eight subfamilies of aphids and three subfamilies of ants were associated. Only two aphid subfamilies and three ant subfamilies were associated in the tropical area. Higher associations were found in the subtropical region with eight aphid subfamilies and four ant subfamilies (Table 3). All collected information was extracted from 21 countries and the aphid-ant species pairs in each country were measured. In the current database, the USA contained a maximum number of species pairs in both temperate and subtropical zones. The second highest number of species pairs was recored in Turkey (Fig. 2). However, equally associated species pairs were documented from subtropical areas of the world. Overall the patterns of aphid-ant interactions in different ecological zones can be seen in the Figure 3.

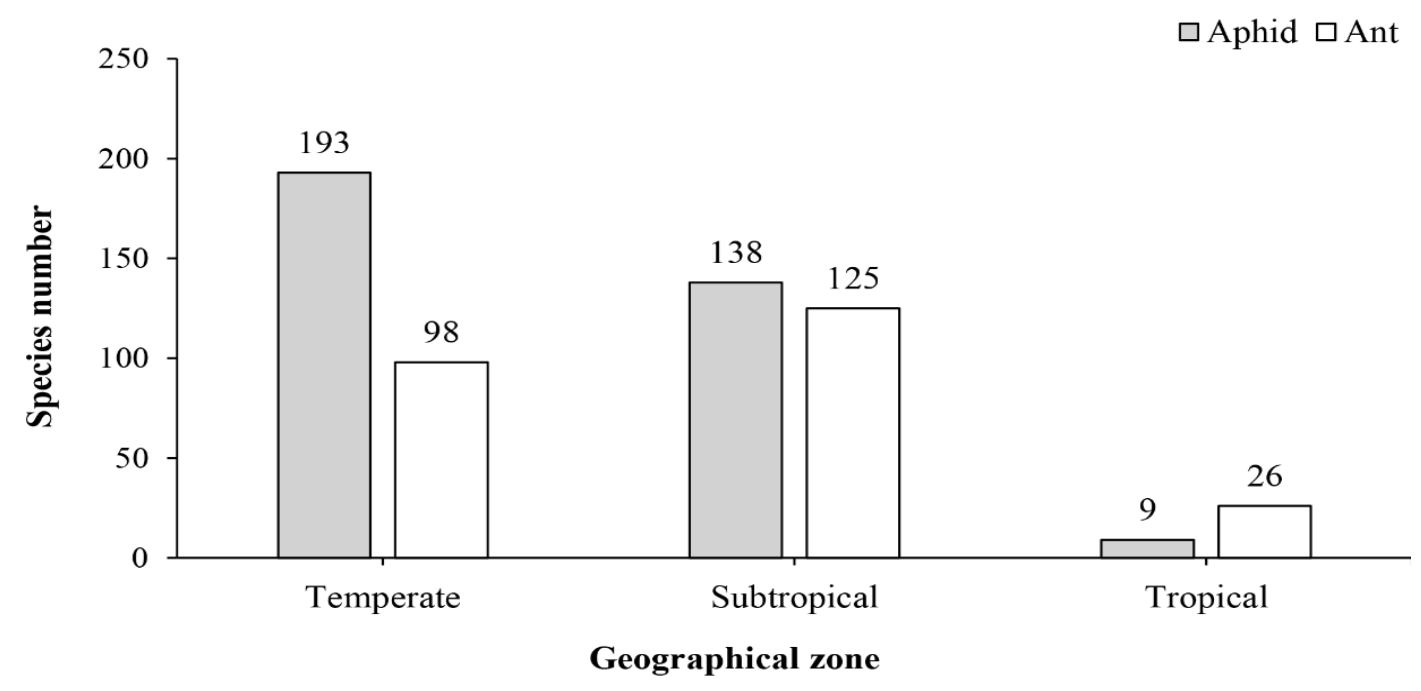

Figure 1. Aphid-ant species pairs in different geographical zones, with the values above bars indicating species numbers of each partner 


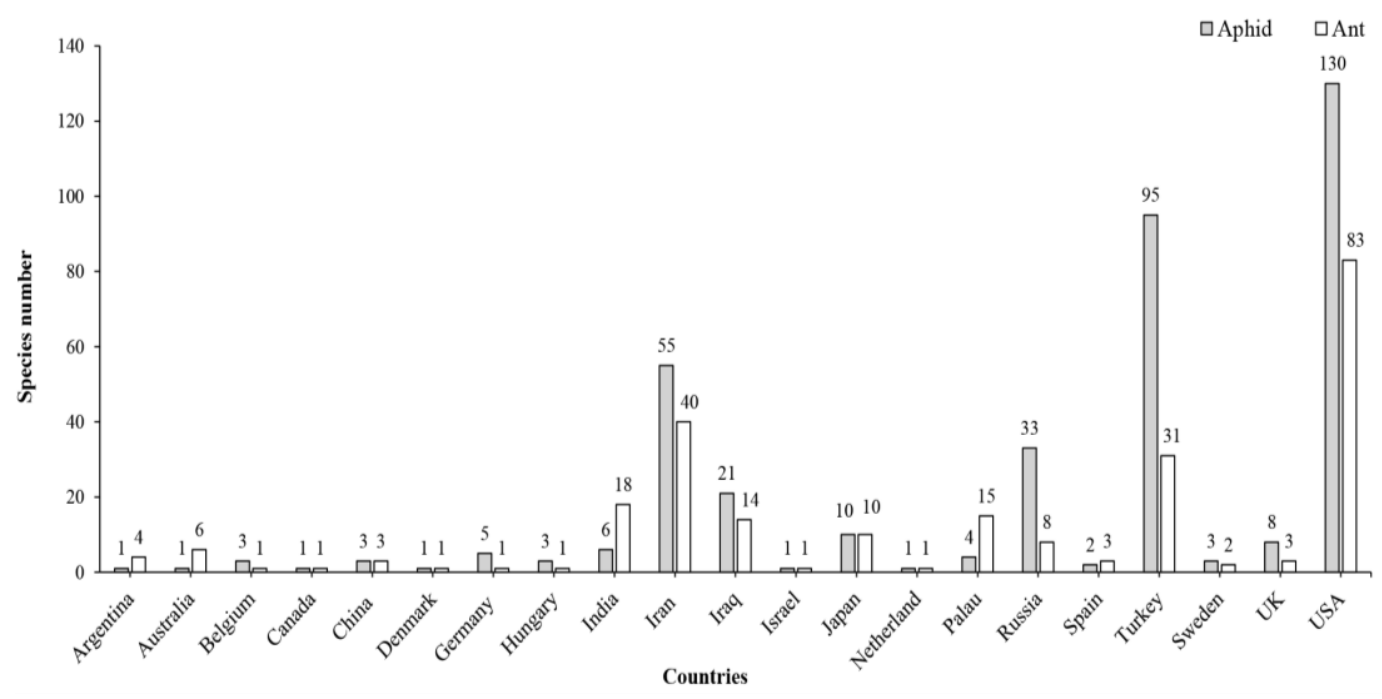

Figure 2. Aphid-ant species pairs in different countries, with the numbers above bars indicating species numbers

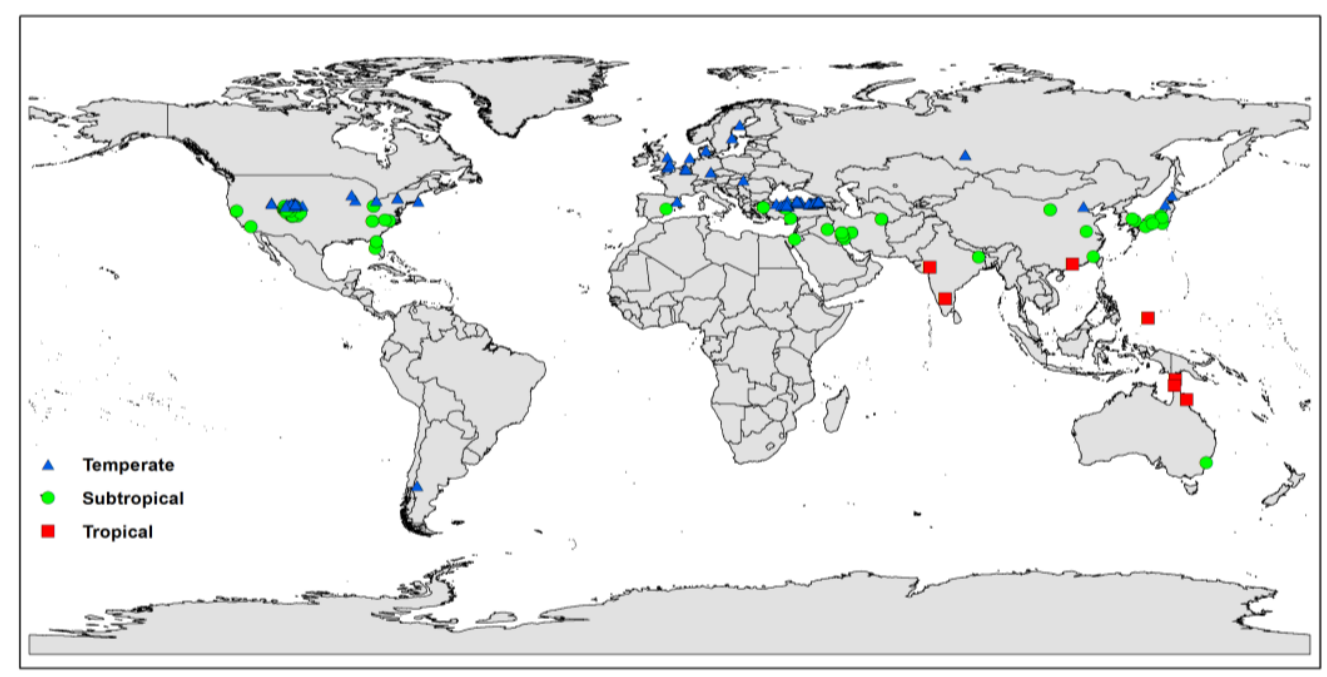

Figure 3. Distribution map of reported aphid-ant associations. The red square, green circle, and blue triangle indicate associations in the tropical, subtropical and temperate regions, respectively

Table 2. The diversity of aphid-associated ants, with the numbers and percentages of ant genera and species in each subfamily. The information of associated aphids for each ant group are also provided

\begin{tabular}{c|c|c|c|c|c|c|c}
\hline \multicolumn{5}{c|}{ Ant } & \multicolumn{3}{c}{ Ant-associated aphid } \\
\hline Subfamily name & Genus & Genus \% & Species & Species \% & Subfamily & Genus & Species \\
\hline Dolichoderinae & 6 & 15.789 & 15 & 7.772 & 5 & 23 & 50 \\
Formicinae & 15 & 39.473 & 122 & 63.212 & 9 & 76 & 261 \\
Myrmicinae & 16 & 42.105 & 55 & 28.497 & 8 & 30 & 84 \\
Pseudomyrmecinae & 1 & 2.631 & 1 & 0.518 & 1 & 1 & 1 \\
Total & 38 & & 193 & & & & \\
\hline
\end{tabular}


Table 3. Associated aphid and ant subfamilies in different geographical zones

\begin{tabular}{c|c|c|c|c|c}
\hline \multicolumn{2}{c|}{ Tropical } & \multicolumn{2}{c|}{ Subtropical } & \multicolumn{2}{c}{ Temperate } \\
\hline $\begin{array}{c}\text { Aphid } \\
\text { subfamily }\end{array}$ & Ant subfamily & $\begin{array}{c}\text { Aphid } \\
\text { subfamily }\end{array}$ & Ant subfamily & $\begin{array}{c}\text { Aphid } \\
\text { subfamily }\end{array}$ & $\begin{array}{c}\text { Ant } \\
\text { subfamily }\end{array}$ \\
\hline Aphidinae & Dolichoderinae & Aphidinae & Dolichoderinae & Anoeciinae & Dolichoderinae \\
Eriosomatinae & Formicinae & Calaphidinae & Formicinae & Aphidinae & Formicinae \\
& Myrmicinae & Chaitophorinae & Myrmicinae & Calaphidinae & Myrmicinae \\
& & Drepanosiphinae & Pseudomyrmecinae & Chaitophorinae & \\
& & Eriosomatinae & & Drepanosiphinae & \\
& & Lachninae & & Eriosomatinae & \\
& & & Lachninae & \\
& & Saltusaphidinae & & Thelaxinae & \\
\hline
\end{tabular}

\section{Discussion}

Our data showed that among the 24 subfamilies of Aphididae (Favret, 2018), only nine are myrmecophilous (Table 1). The aphid subfamily Aphidinae has maximum number of associations with ant species, which corresponds well to the total species diversity of this aphid subfamily. Their maximum associations might be due to some ecological traits and population dynamics (Stadler and Dixon, 2005). A large variety of biological components have been projected to account for population-level variance in aphid-ant interactions including local species abundance and composition of aphids (Horvitz and Schemske, 1984). There are total 23 subfamilies of ants, but only six of them are associated with hemipteran insects (Styrsky and Eubanks, 2007). According to previous statistics, only the subfamilies Dolichoderinae, Formicinae and Myrmicinae harvest honeydew from aphids (Nixon, 1951; Stadler and Dixon, 2005). According to this study, four ant subfamilies, Dolichoderinae, Formicinae, Myrmicinae, and Pseudomyrmecinae (Table 2), are involved in aphid-ant interactions. Styrsky and Eubank (2007) indicated that $89 \%$ of ants from Formicinae and Myrmicinae involved in ant-hemipteran relationships. In the data we complied, it is observed that about $92 \%$ species of ants from Formicinae and Myrmicinae are associated with aphids. According to the Novgorodova and Gavrilyuk (2012) these subfamilies also have maximum trophobiotic interactions with aphids that have a significant effect on aphido-fauna. However, according to the results of this study few species of subfamilies Dolichoderinae and Pseudomyrmecinae were associated in trophobiotic relationships with aphid. The diversity of the mutualistic interaction of subfamily Formicinae might be due to their abundance and ecological dominance. Some subfamilies were less associated with aphids that might be due to less attention paid on these genera of ants.

Diversity and richness of aphid species was higher than ants in the temperate zone (Dixon et al., 1987; Clua et al., 2004), an aphid-ant species ratio of 1.2:1 indicated their abundance in this region (Figure 1). In the temperate region, one aphid species tended by many ant species such as the Novgorodova and Ryabinin (2017) documented that the aphid species Symydobius oblongus, Chaitophorus populeti, Glyphina betulae, Aphis fabae, A. farinosa, A. urticata, Rhopalosiphum padi were associated with 7 to 18 species of ants from temperate areas of Russia. In contrast, ants are exclusively abundant in the tropical zone (Hölldobler and Wilson, 1990; Sarnat et al., 2015). According to current study the aphid-ant species ratios in tropical areas were 1:2.8, 
which indicated that the ants are more than half of the aphid species in these regions. For example, a tropical aphid Cerataphis fransseni attended by various species of ants (Stadler and Dixon, 2005) indicated the dominance of ants in the tropical region. On the other hand, the aphid-ant species ratio was 1.1:1 in subtropical region, which shows that the diversity of species pairs relativity higher in subtropical than the temperate and tropical regions (Figure 1). Most likely the aphids and ants might interact more in subtropical regions which have more favorable conditions for both groups.

The subtropical region consists of a typical warm humid climate with diverse vegetations (e.g. semi-evergreen or semi-deciduous forests, savannah, hardwood forests, pastures, warm temperate moist forests, semi-desert and nemoral deciduous forests) (Prentice et al., 1992; Webb, 2015), which supports the insect and plant biodiversity. Considering aphids are abundant in the temperate zone while ants are most diverse in the tropical, it is suggested that there should be more diverse aphid-ant associations because both groups can interact more in the subtropical zone located between temperate and tropical zones. Geographical species distribution patterns found linear on the borders of temperate and subtropics (Figure 3). There might be two possibilities, 1) the diversity of species pairs higher than other regions, and 2) the studies recorded aphid-ant interactions vastly carried out in these regions. However, the current data may also indicate the research on diversity of aphid-ant interactions is still inadequate, which causes an imbalance of information. Therefore, we suggest more future studies should be undertaken to record more associated species of aphids and ants and then we can understand more about their mutualistic relationships.

\section{Conclusion}

Based on a meta-analysis of currently available records of aphid-ant interactions, this study reports that 284 species belonging to the 83 genera of eight aphid subfamilies have mutualistic association with 193 species belonging to 38 genera of four subfamilies of ants. These species support a total of 972 aphid-ant interactions and 915 ant-aphid interactions (one aphid species may associate with many ant species and vice versa). The aphid genus Aphis has a highest number of associations with ant species while highest number of aphids are associated with the ant genus Formica. Geographically, maximum aphid-ant species pairs were found in subtropical areas of the world. Our results provide a base for identifying general patterns of aphid-ant species pairs in different ecological zones. Besides, more taxonomic and ecological studies are needed to enrich our understanding of the aphid-ant associations.

Acknowledgements. We would like to thank help from Dr. Jun Deng, Taimoor Hassan Farooq, Waqar Ahmed during this work. This work was supported by National Key R\&D Program of China (2016YFE0203100) and Open Fund of Fujian Provincial Key Laboratory of Insect Ecology, Fujian Agriculture and Forestry University.

Author contribution. J.A.S., X. H. conceived and designed the study. J.A.S. performed all the formal analysis. J.L. performed the distribution mapping. J.A.S. and X.Z. collected and compiled the data. X.H. and I.B. reviewed and edited the manuscript.

Conflict of interests. Authors declared that there is no conflict of interests. 


\section{REFERENCES}

[1] Addicott, J. F. (1978): Competition for mutualists: aphids and ants. - Canadian Journal of Zoology 56(10): 2093-2096.

[2] Bascompte, J., Jordano, P. (2007): Plant-animal mutualistic networks: the architecture of biodiversity. - Annu. Rev. Ecol. Evol. Syst. 38: 567-593.

[3] Blackman, R. L., Eastop, V. F. (1984): Aphids on the World's Crops. - Wiley, Chichester, UK.

[4] Boucher, D. H., James, S., Keeler, K. H. (1982): The ecology of mutualism. - Annual Review of Ecology and Systematics 13(1): 315-347.

[5] Bronstein, J. L. (1994): Conditional outcomes in mutualistic interactions. - Trends in Ecology \& Evolution 9(6): 214-217.

[6] Bronstein, J. L. (2009): The evolution of facilitation and mutualism. - Journal of Ecology 97(6): 1160-1170.

[7] Bronstein, J. L., Barbosa, P. (2002): Multitrophic/Multispecies Mutualistic, Multitrophic Level Interactions. - Cambridge University Press, New York.

[8] Buckley, R. (1987): Ant-plant-homopteran interactions. - Advances in Ecological Research 16: 53-85.

[9] Buruchara, R., Mukaruziga, C., Ampofo, K. O. (2010): Bean Disease and Pest Identification and Management. - Centro Internacional de Agricultura Tropical (CIAT), PABRA, Kampala, Uganda, pp. 1-79.

[10] Carabalí-Banguero, D. J., Wyckhuys, K. A. G., Montoya-Lerma, J., Kondo, T., Lundgren, J. G. (2013): Do additional sugar sources affect the degree of attendance of D ysmicoccus brevipes by the fire ant $\mathrm{S}$ olenopsis geminata? - Entomologia Experimentalis et Applicata 148(1): 65-73.

[11] Clua, A., Castro, A. M., Ramos, S., Gimenez, D. O., Vasicek, A., Chidichimo, H. O., Dixon, A. F. G. (2004): The biological characteristics and distribution of the greenbug, Schizaphis graminum, and Russian wheat aphid, Diuraphis noxia (Hemiptera: Aphididae), in Argentina and Chile. - European Journal of Entomology 101(1): 193-198.

[12] Darwin, C. (1859): On the Origin of Species by Means of Natural Selection. - D. Appleton and Company, New York, pp. 83-85.

[13] Delabie, J. H. C. (2001): Trophobiosis between Formicidae and Hemiptera (Sternorrhyncha and Auchenorrhyncha): an overview. - Neotropical Entomology 30(4): 501-516.

[14] Dixon, A. F. G., Kindlmann, P., Leps, J., Holman, J. (1987): Why there are so few species of aphids, especially in the tropics. - The American Naturalist 129(4): 580-592.

[15] Favret, C. (2018): Aphid Species File Ver. 5.0. - <http://Aphid.SpeciesFile.org> (accessed September 15, 2018).

[16] Floate, K. D., Whitham, T. G. (1994): Aphid-ant interaction reduces chrysomelid herbivory in a cottonwood hybrid zone. - Oecologia 97(2): 215-221.

[17] Gilman, S. E., Urban, M. C., Tewksbury, J., Gilchrist, G. W., Holt, R. D. (2010): A framework for community interactions under climate change. - Trends in Ecology \& Evolution 25(6): 325-331.

[18] Heie, O. E. (1994): Why are there so few aphid species in the temperate areas of the southern hemisphere? - European Journal of Entomology 91(1): 127-133.

[19] Herre, E. A., Knowlton, N., Mueller, U. G., Rehner, S. A. (1999): The evolution of mutualisms: exploring the paths between conflict and cooperation. - Trends in Ecology \& Evolution 14(2): 49-53.

[20] Hoeksema, J. D., Bruna, E. M. (2000): Pursuing the big questions about interspecific mutualism: a review of theoretical approaches. - Oecologia 125(3): 321-330.

[21] Hölldobler, B., Wilson, E. O. (1990): The Ants. - Belknap Press of Harvard University Press, Cambridge, MA. 
[22] Horvitz, C. C., Schemske, D. W. (1984), Effects of ants and an ant-tended herbivore on seed production of a neotropical herb. - Ecology 65(5): 1369-1378.

[23] Hosseini, A., Hosseini, M., Katayama, N., Mehrparvar, M. (2017): Effect of ant attendance on aphid population growth and above ground biomass of the aphid's host plant. - European Journal of Entomology 114: 106-112.

[24] Huang, X.-L., Xiang-Yu, J.-G., Ren, S.-S., Zhang, R.-L., Zhang, Y.-P., Qiao, G.-X. (2012): Molecular phylogeny and divergence times of Hormaphidinae (Hemiptera: Aphididae) indicate Late Cretaceous tribal diversification. - Zoological Journal of the Linnean Society 165(1): 73-87.

[25] Ito, F., Gobin, B. (2009): Colony composition and behaviour of a queen and workers in the oriental ectatommine ant Gnamptogenys cribrata (Emery) 1900 in West Java, Indonesia. - Asian Myrmecology 2(1): 103-107.

[26] Jahn, G. C., Beardsley, J. W. (1996): Effects of Pheidole megacephala (Hymenoptera: Formicidae) on survival and dispersal of Dysmicoccus neobrevipes (Homoptera: Pseudococcidae). - Journal of Economic Entomology 89(5): 1124-1129.

[27] Katayama, N., Suzuki, N. (2003): Changes in the use of extrafloral nectaries of Vicia faba (Leguminosae) and honeydew of aphids by ants with increasing aphid density. - Annals of the Entomological Society of America, BioOne 96(4): 579-584.

[28] Mittler, T. E. (1958): The excretion of honey-dew by Tuberolachnus salignus (Gmelin) (Homoptera: Aphididae). - Proceedings of the Royal Entomological Society of London. Series A, General Entomology 33: 49-55.

[29] Moya-Ragoza, G., Nault, L. R. (2000): Obligatory mutualism between Dalbulus quinquenotatus (Homoptera: Cicadellidae) and attendant ants. - Annals of the Entomological Society of America 93(4): 929-940.

[30] Nixon, G. E. J. (1951): The Association of Ants with Aphids and Coccids. - Commonw. Inst. Ent., London.

[31] Novgorodova, T. A. (2002): Study of adaptations of aphids (Homoptera, Aphidinea) to ants: comparative analysis of myrmecophilous and nonmyrmecophilous species. Entomological Review 82(5): 569-576.

[32] Novgorodova, T. A. (2005): Ant-aphid interactions in multispecies ant communities: Some ecological and ethological aspects. - European Journal of Entomology 102(3): 495-501.

[33] Novgorodova, T. A. (2015): Organization of honeydew collection by foragers of different species of ants (Hymenoptera: Formicidae): effect of colony size and species specificity. - European Journal of Entomology 112(4): 688.

[34] Novgorodova, T. A., Gavrilyuk, A. V. (2012): The degree of protection different ants (Hymenoptera: Formicidae) provide aphids (Hemiptera: Aphididae) against aphidophages. - European Journal of Entomology 109(2): 187-196.

[35] Novgorodova, T. A., Ryabinin, A. S. (2017): Ant-aphid relations in the south of western Siberia (Hymenoptera: Formicidae; Hemiptera: Aphididae). - Arthropod-Plant Interactions. DOI: 10.1007/s11829-017-9584-7.

[36] Philpott, S. M., Armbrecht, I. (2006): Biodiversity in tropical agroforests and the ecological role of ants and ant diversity in predatory function. - Ecological Entomology 31(4): 369-377.

[37] Prentice, I. C., Cramer, W., Harrison, S. P., Leemans, R., Monserud, R. A., Solomon, A. M. (1992): Special paper: a global biome model based on plant physiology and dominance, soil properties and climate. - Journal of Biogeography 19(2): 117.

[38] Rico-Gray, V., Castro, G. (1996): Effect of an ant-aphid interaction on the reproductive fitness of Paullinia fuscecens (Sapindaceae). - The Southwestern Naturalist 41: 434-440.

[39] Sarnat, E. M., Fischer, G., Guénard, B., Economo, E. P. (2015): Introduced pheidole of the world: taxonomy, biology and distribution. - ZooKeys 2015(543): 1-109.

[40] Stadler, B., Dixon, A. F. G. (1998): Costs of ant attendance for aphids. - Journal of Animal Ecology 67(3): 454-459. 
[41] Stadler, B., Dixon, A. F. G. (2005): Ecology and evolution of aphid-ant interactions. Annual Review of Ecology, Evolution, and Systematics 36(1): 345-372.

[42] Styrsky, J. D., Eubanks, M. D. (2007): Ecological consequences of interactions between ants and honeydew-producing insects. Proceedings. - Biological Sciences/The Royal Society 274(1607): 151-64.

[43] Tylianakis, J. M., Didham, R. K., Bascompte, J., Wardle, D. A. (2008): Global change and species interactions in terrestrial ecosystems. - Ecology Letters 11(12): 1351-1363.

[44] Ward, P. S. (ed.) (2013): AntWeb: Ants of California. http://www.AntWeb.org/california.jsp (accessed 15 October 2018).

[45] Way, M. J. (1963): Mutualism between ants and honeydew-producing Homoptera. Annual Review of Entomology 8(1): 307-344.

[46] Way, M. J., Khoo, K. C. (1992): Role of ants in pest management. - Annual Review of Entomology 37(1): 479-503.

[47] Webb, L. J. (2015): Environmental relationships of the structural types of australian rain forest vegetation. - Ecology 49(2): 296-311.

\section{APPENDIX}

Table A1. Species pairs of mutualistic aphids and ants

\begin{tabular}{|c|c|c|}
\hline Aphid & Associated ant & Reference \\
\hline \multicolumn{3}{|l|}{ Subfamily Aphidinae } \\
\hline \multicolumn{3}{|l|}{ Tribe Aphidini } \\
\hline Aphis acetosae & Formica cunicularia & Mortazavi et al. (2015) \\
\hline Aphis aiddletonii & Formica argentea & Jones (1927) \\
\hline \multirow{19}{*}{ Aphis asclepiadis } & Camponotus vicinus & Jones (1927) \\
\hline & Formica argentea & Jones (1927) \\
\hline & Formica aserva & Addicott (1979a) \\
\hline & Formica canadensis & Jones (1927) \\
\hline & Formica cinerea & Jones (1927), Addicott (1979a) \\
\hline & Formica fusca & Jones (1927), Addicott (1979a) \\
\hline & Formica integroides & Addicott (1979a) \\
\hline & Formica lasioides & Addicott (1979a) \\
\hline & Formica lepida & Jones (1927) \\
\hline & Formica montana & Jones (1927) \\
\hline & Formica neorufibarbis & Addicott (1979a) \\
\hline & Formica obscuripes & Addicott (1979a) \\
\hline & Formica opaciventris & Jones (1927) \\
\hline & Formica podzolica & $\begin{array}{l}\text { Mooney and Agrawal (2008), Nielsen et al. } \\
\text { (2009), Nielsen et al. (2009), Abdala-Roberts } \\
\text { et al. (2012) }\end{array}$ \\
\hline & Myrmica incompleta & Jones $(1927)$ \\
\hline & Myrmica scabrinodis & Jones (1927) \\
\hline & Myrmica whymperi & Jones (1927) \\
\hline & Pheidole bicarinata & Jones (1927) \\
\hline & Tapinoma sessile & Jones (1927), Addicott (1979a) \\
\hline Aphis aurantii & Formica cinereofusca & Akyildirim et al. (2014) \\
\hline
\end{tabular}




\begin{tabular}{|c|c|c|}
\hline & Lasius alienus & Akyildirim et al. (2014) \\
\hline & Lasius turcicus & Akyildirim et al. (2014) \\
\hline & Plagiolepis taurica & Akyildirim et al. (2014) \\
\hline Aphis brassicae & Pheidole sp. & Kataria and Kumar (2013) \\
\hline \multirow{3}{*}{ Aphis brohmeri } & Camponotus saxatilis & Novgorodova (2005) \\
\hline & Formica aquilonia & Novgorodova (2005) \\
\hline & Formica polyctena & Novgorodova (2005) \\
\hline \multirow{3}{*}{ Aphis brotericola } & Camponotus aethiops & Özdemir et al. (2008) \\
\hline & Camponotus piceus & Özdemir et al. (2008) \\
\hline & Formica cunicularia & Akyürek et al. (2016) \\
\hline Aphis brunellae & Lasius turcicus & Akyürek et al. (2016) \\
\hline Aphis chloris & Camponotus piceus & Özdemir et al. (2008) \\
\hline Aphis cinara & Formica lugubris & Sudd (1983) \\
\hline \multirow{5}{*}{ Aphis clerodendri } & Camponotus vitreus & Carver et al. (2003) \\
\hline & Iridomyrmex hartmeyeri & Carver et al. (2003) \\
\hline & Notoncus capitatus & Carver et al. (2003) \\
\hline & Paratrechina vaga & Carver et al. (2003) \\
\hline & Technomyrmex albipes & Carver et al. (2003) \\
\hline \multirow{5}{*}{ Aphis coreopsidis } & Crematogaster lineolata & Favret et al. (2010) \\
\hline & Formica subsericea & Favret et al. (2010) \\
\hline & Linepithema humile & Altfeld and Stiling (2009) \\
\hline & Paratrechina longicornis & Favret et al. (2010) \\
\hline & Paratrechina parvula & Favret et al. (2010) \\
\hline Aphis cornifoliae & Prenolepis imparis & Favret et al. (2010) \\
\hline Aphis craccae & Lasius niger & Novgorodova (2005) \\
\hline \multirow{19}{*}{ Aphis craccivora } & Camponotus compressus & $\begin{array}{l}\text { Kataria and Kumar (2013), Rakhshan and } \\
\text { Ahmad (2015) }\end{array}$ \\
\hline & Camponotus invidus & Rakhshan and Ahmad (2015) \\
\hline & Camponotus kurdistanicus & Stary (1969) \\
\hline & Camponotus libanicus & Shiran et al. (2013) \\
\hline & Camponotus oasium & Shiran et al. (2013) \\
\hline & Camponotus piceus & Özdemir et al. (2008) \\
\hline & Cataglyphis aenescens & Özdemir et al. (2008) \\
\hline & Crematogaster antaris & Shiran et al. (2013) \\
\hline & Crematogaster inermis & Mortazavi et al. (2015) \\
\hline & Crematogaster schmidti & Mortazavi et al. (2015) \\
\hline & Crematogaster subdentata & Mortazavi et al. (2015) \\
\hline & Formica aquilonia & Novgorodova (2005) \\
\hline & Formica cinereofusca & Akyildirim et al. (2014) \\
\hline & Formica cunicularia & Özdemir et al. (2008), Mortazavi et al. (2015) \\
\hline & Formica fusca & Akyürek et al. (2016) \\
\hline & Formica glauca & Özdemir et al. (2008) \\
\hline & Formica polyctena & Novgorodova (2005) \\
\hline & Formica pratensis & Novgorodova (2005) \\
\hline & Lasius brunneus & Akyürek et al. (2016) \\
\hline
\end{tabular}




\begin{tabular}{|c|c|c|}
\hline & Lasius japonicus & Suzuki (2004), Katayama et al. (2013) \\
\hline & Lasius niger & $\begin{array}{c}\text { Novgorodova (2005), Kataria and Kumar } \\
\text { (2013) }\end{array}$ \\
\hline & Lasius paralienus & Özdemir et al. (2008), Shiran et al. (2013) \\
\hline & Lasius turcicus & $\begin{array}{l}\text { Akyildirim et al. (2014), Mortazavi et al. } \\
\text { (2015) }\end{array}$ \\
\hline & Lepisiota bipartite & Shiran et al. (2013) \\
\hline & Lepisiota frauenfeldi & Rakhshan and Ahmad (2015) \\
\hline & Meranoplus bicolor & Rakhshan and Ahmad (2015) \\
\hline & Monomorium destructor & Shiran et al. (2013) \\
\hline & Monomorium latinode & Rakhshan and Ahmad (2015) \\
\hline & Monomorium mayri & Shiran et al. (2013) \\
\hline & Monomorium minimum & Kataria and Kumar (2013) \\
\hline & Monomorium nitidiventre & Mortazavi et al. (2015) \\
\hline & Monomorium pharaonis & Rakhshan and Ahmad (2015) \\
\hline & Nylanderia fulva & Sharma and Buss (2013) \\
\hline & Paratrechina longicornis & $\begin{array}{c}\text { Shiran et al. (2013), Rakhshan and Ahmad } \\
\text { (2015) }\end{array}$ \\
\hline & Pheidole teneriffana & Shiran et al. (2013) \\
\hline & Plagiolepis pallescens & Özdemir et al. (2008), Shiran et al. (2013) \\
\hline & Plagiolepis pygmaea & Mortazavi et al. (2015) \\
\hline & Polyrhachis hauxwelli & Rakhshan and Ahmad (2015) \\
\hline & Polyrhachis lacteipennis & Shiran et al. (2013) \\
\hline & Pristomyrmex punctatus & Katayama et al. (2013) \\
\hline & Tapinoma erraticum & Mortazavi et al. (2015) \\
\hline & Tapinoma simrothi & Stary (1969), Shiran et al. (2013) \\
\hline & Tetramorium chefteki & Özdemir et al. (2008), Mortazavi et al. (2015) \\
\hline & Tetramorium forte & Özdemir et al. (2008) \\
\hline & Tetramorium tsushimae & $\begin{array}{l}\text { Suzuki (2004), Katayama et al. (2013), } \\
\text { Hayashi et al. (2015), Hayashi et al. (2016) }\end{array}$ \\
\hline & Tetraponera rufonigra & Rakhshan and Ahmad (2015) \\
\hline \multirow{2}{*}{ Aphis davletshinae } & Pheidole pallidula & Shiran et al. (2013) \\
\hline & Tapinoma simrothi & Shiran et al. (2013) \\
\hline Aphis esulae & Formica fusca & Akyürek et al. (2016) \\
\hline Aphis euphorbiae & Camponotus piceus & Özdemir et al. (2008) \\
\hline \multirow{2}{*}{ Aphis evonymi } & Formica aquilonia & Novgorodova (2005) \\
\hline & Formica polyctena & Novgorodova (2005) \\
\hline \multirow{9}{*}{ Aphis fabae } & Camponotus aethiops & Akyürek et al. (2016) \\
\hline & Camponotus compressus & Kataria and Kumar (2013) \\
\hline & Camponotus piceus & Özdemir et al. (2008) \\
\hline & Camponotus saxatilis & Novgorodova (2005) \\
\hline & Cataglyphis lividus & Shiran et al. (2013) \\
\hline & Cremastogaster scutellaris & Akyürek et al. (2016) \\
\hline & Crematogaster lineolata & Favret et al. (2010) \\
\hline & Formica aquilonia & Novgorodova (2005) \\
\hline & Formica cinereofusca & Akyildirim et al. (2014) \\
\hline
\end{tabular}




\begin{tabular}{|c|c|c|}
\hline & Formica cunicularia & Akyürek et al. (2016) \\
\hline & Formica fusca & Akyildirim et al. (2014) \\
\hline & Formica polyctena & Novgorodova (2005) \\
\hline & Lasius alienus & Akyildirim et al. (2014), Akyürek et al. (2016) \\
\hline & Lasius brunneus & Akyürek et al. (2016) \\
\hline & Lasius emarginatus & Akyildirim et al. (2014) \\
\hline & Lasius niger & $\begin{array}{l}\text { Liepert and Dettner (1993), Volkl et al. (1999), } \\
\text { Offenberg (2001), Woodring et al. (2004), } \\
\text { Fischer et al. (2005), Detrain et al. (2010), } \\
\text { Sanders et al. (2010), Tegelaar et al. (2012), } \\
\text { Tegelaar et al. (2013), Fischer et al. (2015), } \\
\text { Tegelaar (2015), Vantaux et al. (2015) }\end{array}$ \\
\hline & Lasius paralienus & Özdemir et al. (2008), Shiran et al. (2013) \\
\hline & Lasius turcicus & Akyildirim et al. (2014), Akyürek et al. (2016) \\
\hline & Lepisiota bipartite & Shiran et al. (2013) \\
\hline & Lepisiota nigra & Mortazavi et al. (2015) \\
\hline & Monomorium libanicum & Shiran et al. (2013) \\
\hline & Monomorium mayri & Shiran et al. (2013) \\
\hline & Monomorium minimum & Kataria and Kumar (2013) \\
\hline & Monomorium qarahe & Shiran et al. (2013) \\
\hline & Myrmica ruginodis & Akyildirim et al. (2014) \\
\hline & Plagiolepis pallescens & Shiran et al. (2013) \\
\hline & Plagiolepis pygmaea & Akyürek et al. (2016) \\
\hline & Prenolepis imparis & Favret et al. (2010) \\
\hline & Tapinoma erraticum & Mortazavi et al. (2015) \\
\hline & Tapinoma simrothi & Stary (1969), Shiran et al. (2013) \\
\hline & Tetramorium caespitum & Shiran et al. (2013), Akyildirim et al. (2014) \\
\hline & Tetramorium tsushimae & Hayashi et al. (2015) \\
\hline & Formica glauca & Özdemir et al. (2008) \\
\hline \multirow{8}{*}{ Aphis farinosa } & Crematogaster antaris & Shiran et al. (2013) \\
\hline & Formica cinereofusca & Akyildirim et al. (2014) \\
\hline & Formica fusca & Jones (1927) \\
\hline & Formica gagates & Akyildirim et al. (2014) \\
\hline & Formica integroides & Addicott (1978) \\
\hline & Lasius turcicus & Akyildirim et al. (2014) \\
\hline & Myrmica whymperi & Jones (1927) \\
\hline & Tetramorium caespitum & Akyildirim et al. (2014) \\
\hline \multirow{3}{*}{ Aphis forbesi } & Formica cinerea & Jones (1927) \\
\hline & Myrmecina graminicola & Jones (1927) \\
\hline & Myrmica scabrinodis & Jones (1927) \\
\hline \multirow{6}{*}{ Aphis frangulae } & Camponotus oasium & Shiran et al. (2013) \\
\hline & Cataglyphis nodus & Shiran et al. (2013) \\
\hline & Crematogaster antaris & Shiran et al. (2013) \\
\hline & Lasius paralienus & Shiran et al. (2013) \\
\hline & Lepisiota bipartite & Shiran et al. (2013) \\
\hline & Monomorium destructor & Shiran et al. (2013) \\
\hline
\end{tabular}




\begin{tabular}{|c|c|c|}
\hline & Monomorium libanicum & Shiran et al. (2013) \\
\hline & Monomorium mayri & Shiran et al. (2013) \\
\hline & Monomorium qarahe & Shiran et al. (2013) \\
\hline & Pheidole pallidula & Shiran et al. (2013) \\
\hline & Plagiolepis pallescens & Shiran et al. (2013) \\
\hline & Polyrhachis lacteipennis & Shiran et al. (2013) \\
\hline & Tapinoma simrothi & Shiran et al. (2013) \\
\hline \multirow{3}{*}{ Aphis galliiscabri } & Formica glauca & Özdemir et al. (2008) \\
\hline & Lasius alienus & Özdemir et al. (2008) \\
\hline & Lasius paralienus & Özdemir et al. (2008) \\
\hline Aphis gerardianae & Plagiolepis pygmaea & Akyürek et al. (2016) \\
\hline \multirow{32}{*}{ Aphis gossypii } & Anoplolepis gracilipes & Idechiil et al. (2007) \\
\hline & Camponotus compressus & $\begin{array}{l}\text { Verghese and Tandon (1987), Kataria and } \\
\text { Kumar (2013), Lokeshwari et al. (2015) }\end{array}$ \\
\hline & Camponotus parius & Lokeshwari et al. (2015) \\
\hline & Camponotus reticulatus & Idechiil et al. (2007) \\
\hline & Cataglyphis nodus & Stary (1969) \\
\hline & Crematogaster inermis & Mortazavi et al. (2015) \\
\hline & Crematogaster lineolata & Favret et al. (2010) \\
\hline & Formica argentea & Jones (1927) \\
\hline & Formica cinerea & Jones (1927) \\
\hline & Formica cinereofusca & Akyildirim et al. (2014) \\
\hline & Formica fusca & Jones (1927) \\
\hline & Iridomyrmex anceps & Idechiil et al. (2007) \\
\hline & Lasius alienus & Akyildirim et al. (2014), Akyürek et al. (2016) \\
\hline & Lasius emarginatus & Akyildirim et al. (2014) \\
\hline & Lasius niger & Kaneko (2003) \\
\hline & Lasius paralienus & Özdemir et al. (2008) \\
\hline & Lasius turcicus & Akyildirim et al. (2014), Akyürek et al. (2016) \\
\hline & Lepisiota nigra & Mortazavi et al. (2015) \\
\hline & Linepithema humile & $\begin{array}{l}\text { Powell and Silverman (2010), Tena et al. } \\
\text { (2013), LeVan and Holway (2015) }\end{array}$ \\
\hline & $\begin{array}{l}\text { Lophomyrmex } \\
\text { quadrispinosus }\end{array}$ & Lokeshwari et al. (2015) \\
\hline & Monomorium floricola & Idechiil et al. (2007) \\
\hline & Monomorium minimum & Kataria and Kumar (2013) \\
\hline & Monomorium monomorium & Idechiil et al. (2007) \\
\hline & Myrmica incompleta & Jones (1927) \\
\hline & Myrmicaria brunnea & Lokeshwari et al. (2015) \\
\hline & Paratrechina bourbonica & Idechiil et al. (2007) \\
\hline & Paratrechina longicornis & Idechiil et al. (2007), Lokeshwari et al. (2015) \\
\hline & Pheidole fervens & Idechiil et al. (2007) \\
\hline & Plagiolepis pygmaea & Akyürek et al. (2016) \\
\hline & Pristomyrmex pungens & Kaneko (2003) \\
\hline & Solenopsis geminata & Idechiil et al. (2007), Lokeshwari et al. (2015) \\
\hline & Tapinoma erraticum & Mortazavi et al. (2015) \\
\hline
\end{tabular}




\begin{tabular}{|c|c|c|}
\hline & Tapinoma indicum & Lokeshwari et al. (2015) \\
\hline & Tapinoma melanocephalum & Idechiil et al. (2007), Lokeshwari et al. (2015) \\
\hline & Tapinoma sessile & Powell and Silverman (2010) \\
\hline & Tapinoma simrothi & Stary (1969) \\
\hline & Technomyrmex albipes & Idechiil et al. (2007), Lokeshwari et al. (2015) \\
\hline & Tetramorium bicarinatum & Idechiil et al. (2007) \\
\hline & Tetramorium caespitum & Akyildirim et al. (2014) \\
\hline \multirow{3}{*}{ Aphis helianthi } & Formica fusca & Mooney et al. (2015) \\
\hline & Formica rufa & Mooney et al. (2015) \\
\hline & Tapinoma sessile & Mooney et al. (2015) \\
\hline Aphis hermistonii & Formica fusca & Jones (1927) \\
\hline Aphis hillerislambersi & Lasius brunneus & Akyürek et al. (2016) \\
\hline \multirow{2}{*}{ Aphis idaei } & Lasius alienus & Stary (1969) \\
\hline & Lasius niger & Novgorodova (2005) \\
\hline Aphis illinoisensis & Myrmica punctiventris & Favret et al. (2010) \\
\hline Aphis impatientis & Lasius emarginatus & Akyildirim et al. (2014) \\
\hline \multirow{4}{*}{ Aphis jacobaeae } & Formica aquilonia & Novgorodova (2005) \\
\hline & Formica polyctena & Novgorodova (2005) \\
\hline & Lasius niger & $\begin{array}{c}\text { Vrieling et al. (1991), Müller and Godfray } \\
\text { (1999) }\end{array}$ \\
\hline & Myrmica ruginodis & Müller and Godfray (1999) \\
\hline Aphis longituba & Lasius emarginatus & Akyildirim et al. (2014) \\
\hline \multirow{9}{*}{ Aphis lugentis } & Camponotus herculeanus & Jones (1927) \\
\hline & Camponotus modoc & Jones (1927) \\
\hline & Dorymyrmex pyramicus & Jones (1927) \\
\hline & Formica argentea & Jones (1927) \\
\hline & Formica canadensis & Jones (1927) \\
\hline & Formica efiniventris & Jones (1927) \\
\hline & Formica fusca & Jones (1927) \\
\hline & Myrmica punctiventris & Favret et al. (2010) \\
\hline & Tapinoma sessile & Favret et al. (2010) \\
\hline Aphis maculatae & Formica fusca & Jones (1927) \\
\hline Aphis maidiradicis & Lasius alienus & Forbes (1906) \\
\hline \multirow{7}{*}{ Aphis medicaginis } & Formica argentea & Jones (1927) \\
\hline & Formica canadensis & Jones (1927) \\
\hline & Formica cinerea & Jones (1927) \\
\hline & Formica fusca & Jones (1927) \\
\hline & Formica opaciventris & Jones (1927) \\
\hline & Formica rufa & Jones (1927) \\
\hline & Myrmica incompleta & Jones (1927) \\
\hline Aphis middletonii & Lasius brunneus & Akyürek et al. (2016) \\
\hline Aphis molluginis & Lasius alienus & Akyildirim et al. (2014) \\
\hline \multirow{3}{*}{ Aphis nasturtii } & Formica cinereofusca & Akyildirim et al. (2014) \\
\hline & Formica cunicularia & Akyürek et al. (2016) \\
\hline & Lasius alienus & Özdemir et al. (2008) \\
\hline
\end{tabular}




\begin{tabular}{|c|c|c|}
\hline & Lasius brunneus & Akyürek et al. (2016) \\
\hline & Lasius emarginatus & Akyildirim et al. (2014) \\
\hline & Lepisiota bipartite & Shiran et al. (2013) \\
\hline & Tapinoma simrothi & Shiran et al. (2013) \\
\hline \multirow{9}{*}{ Aphis nerii } & Camponotus compressus & Kataria and Kumar (2013) \\
\hline & Iridomyrmex humilis & Bristow (1991) \\
\hline & Lepisiota bipartite & Shiran et al. (2013) \\
\hline & Linepithema humile & Pringle et al. (2014) \\
\hline & Monomorium gracillimum & Stary (1969) \\
\hline & Monomorium mayri & Shiran et al. (2013) \\
\hline & Monomorium minimum & Kataria and Kumar (2013) \\
\hline & Pheidole pallidula & Stary (1969) \\
\hline & Tapinoma simrothi & Shiran et al. (2013) \\
\hline \multirow{2}{*}{ Aphis oenotherae } & Formica fusca & Jones (1927) \\
\hline & Myrmica whymperi & Jones (1927) \\
\hline Aphis oregonensis & Formica montana & Jones (1927) \\
\hline Aphis parietariae & Lepisiota bipartite & Shiran et al. (2013) \\
\hline Aphis polygonata & Monomorium mayri & Shiran et al. (2013) \\
\hline \multirow{15}{*}{ Aphis pomi } & Cremastogaster scutellaris & Akyürek et al. (2016) \\
\hline & Formica aquilonia & Novgorodova (2005) \\
\hline & Formica canadensis & Jones (1927) \\
\hline & Formica cinerea & Jones (1927) \\
\hline & Formica cunicularia & Novgorodova (2005) \\
\hline & Formica fusca & Jones (1927) \\
\hline & Formica polyctena & Novgorodova (2005) \\
\hline & Formica rufibarbis & Akyildirim et al. (2014) \\
\hline & Lasius alienus & Akyürek et al. (2016) \\
\hline & Lasius brunneus & Akyürek et al. (2016) \\
\hline & Lasius niger & Novgorodova (2005) \\
\hline & Lasius turcicus & Akyürek et al. (2016) \\
\hline & Myrmica incompleta & Jones (1927) \\
\hline & Tapinoma erraticum & Mortazavi et al. (2015) \\
\hline & Tapinoma simrothi & Stary (1969) \\
\hline Aphis populicola & Myrmica incompleta & Jones (1927) \\
\hline \multirow{3}{*}{ Aphis pseudocardui } & Crematogaster inermis & Mortazavi et al. (2015) \\
\hline & Crematogaster schmidti & Mortazavi et al. (2015) \\
\hline & Lasius alienus & Akyürek et al. (2016) \\
\hline \multirow{2}{*}{ Aphis punicae } & Lepisiota bipartite & Shiran et al. (2013) \\
\hline & Tapinoma simrothi & Stary (1969) \\
\hline \multirow{6}{*}{ Aphis rumicis } & Lasius paralienus & Özdemir et al. (2008) \\
\hline & Tetramorium chefteki & Özdemir et al. (2008) \\
\hline & Tetramorium forte & Özdemir et al. (2008) \\
\hline & Myrmica incompleta & Jones (1927) \\
\hline & Myrmica whymperi & Jones (1927) \\
\hline & Tapinoma simrothi & Shiran et al. (2013) \\
\hline
\end{tabular}




\begin{tabular}{|c|c|c|}
\hline \multirow{12}{*}{ Aphis salicariae } & Camponotus herculeanus & Addicott (1979a) \\
\hline & Formica aserva & Addicott (1979a) \\
\hline & Formica cinerea & Addicott (1979a) \\
\hline & Formica fusca & Addicott (1979a), Mooney et al. (2015) \\
\hline & Formica integroides & Addicott (1979a) \\
\hline & Formica lasioides & Addicott (1979a) \\
\hline & Formica neorufibarbis & Addicott (1979a) \\
\hline & Formica obscuripes & Addicott (1979a) \\
\hline & Formica puberula & Addicott (1979a) \\
\hline & Formica rufa & Mooney et al. (2015) \\
\hline & Tapinoma erraticum & Mortazavi et al. (2015) \\
\hline & Tapinoma sessile & Addicott (1979a), Mooney et al. (2015) \\
\hline \multirow{3}{*}{ Aphis salviae } & Camponotus aethiops & Özdemir et al. (2008) \\
\hline & Camponotus piceus & Özdemir et al. (2008) \\
\hline & Tetramorium caespitum & Akyürek et al. (2016) \\
\hline \multirow{2}{*}{ Aphis sambuci } & Formica cinereofusca & Akyildirim et al. (2014) \\
\hline & Lasius turcicus & Akyildirim et al. (2014) \\
\hline Aphis sambucifolii & Formica argentea & Jones (1927) \\
\hline Aphis serpylli & Formica cinereofusca & Akyildirim et al. (2014) \\
\hline \multirow{2}{*}{ Aphis solanella } & Formica cunicularia & Akyürek et al. (2016) \\
\hline & Lasius alienus & Akyürek et al. (2016) \\
\hline \multirow{12}{*}{ Aphis spiraecola } & Camponotus lateralis & Akyürek et al. (2016) \\
\hline & Cremastogaster scutellaris & Akyürek et al. (2016) \\
\hline & Formica cinereofusca & Akyildirim et al. (2014) \\
\hline & Formica cunicularia & Akyürek et al. (2016) \\
\hline & Formica rufibarbis & Akyürek et al. (2016) \\
\hline & Lasius brunneus & Akyürek et al. (2016) \\
\hline & Lasius emarginatus & Akyildirim et al. (2014) \\
\hline & Lasius turcicus & Akyildirim et al. (2014), Akyürek et al. (2016) \\
\hline & Linepithema humile & Tena et al. (2013) \\
\hline & Plagiolepis pygmaea & Akyürek et al. (2016) \\
\hline & Plagiolepis taurica & Akyildirim et al. (2014) \\
\hline & Tetramorium caespitum & Akyürek et al. (2016) \\
\hline \multirow{2}{*}{ Aphis subnitidae } & Formica aquilonia & Novgorodova (2005) \\
\hline & Formica polyctena & Novgorodova (2005) \\
\hline Aphis ulmariae & Formica argentea & Jones (1927) \\
\hline \multirow{5}{*}{ Aphis umbrella } & Lasius paralienus & Shiran et al. (2013) \\
\hline & Lepisiota bipartite & Shiran et al. (2013) \\
\hline & Pheidole pallidula & Shiran et al. (2013) \\
\hline & Pheidole teneriffana & Shiran et al. (2013) \\
\hline & Tapinoma simrothi & Shiran et al. (2013) \\
\hline Aphis urticata & Lasius alienus & Akyürek et al. (2016) \\
\hline \multirow{3}{*}{ Aphis valerianae } & Camponotus herculeanus & Jones (1927) \\
\hline & Camponotus vicinus & Jones (1927) \\
\hline & Formica canadensis & Jones (1927) \\
\hline
\end{tabular}




\begin{tabular}{|c|c|c|}
\hline & Formica podzolica & Petry et al. (2012) \\
\hline & Formica rufa & Jones (1927) \\
\hline Aphis vandergooti & Lasius niger & Woodring et al. (2004) \\
\hline \multirow{11}{*}{ Aphis varians } & Camponotus herculeanus & Addicott (1979a) \\
\hline & Formica aserva & Addicott (1979a) \\
\hline & Formica cinerea & $\begin{array}{c}\text { Addicott (1978), Addicott (1979a), Breton and } \\
\text { Addicott (1992) }\end{array}$ \\
\hline & Formica fusca & $\begin{array}{c}\text { Addicott (1978), Addicott (1979a), Mooney et } \\
\text { al. (2015) }\end{array}$ \\
\hline & Formica integroides & Addicott (1978), Addicott (1979a) \\
\hline & Formica lasioides & Addicott (1978), Addicott (1979a) \\
\hline & Formica neorufibarbis & Addicott (1979a) \\
\hline & Formica obscuripes & Addicott (1978), Addicott (1979a) \\
\hline & Formica puberula & Addicott (1979a) \\
\hline & Formica rufa & Mooney et al. (2015) \\
\hline & Tapinoma sessile & Addicott (1979a), Mooney et al. (2015) \\
\hline \multirow{2}{*}{ Aphis verbasci } & Formica cinereofusca & Akyildirim et al. (2014) \\
\hline & Lasius alienus & Özdemir et al. (2008), Akyürek et al. (2016) \\
\hline Aphis vernoniae & Nylanderia fulva & Sharma and Buss (2013) \\
\hline \multirow{2}{*}{ Aphis viburni } & Formica aquilonia & Novgorodova (2005) \\
\hline & Lasius niger & Novgorodova (2005) \\
\hline Brachyonguis harmalae & Tapinoma simrothi & Shiran et al. (2013) \\
\hline $\begin{array}{l}\text { Brachyonguis } \\
\text { tamaricophilus }\end{array}$ & Tapinoma simrothi & Shiran et al. (2013) \\
\hline Brachyunguis tamaricis & Formica cinereofusca & Akyildirim et al. (2014) \\
\hline Brachyunguis tetrapteralis & Formica fusca & Jones (1927) \\
\hline \multirow{2}{*}{ Braggia eriogoni } & Formica argentea & Jones (1927) \\
\hline & Formica fusca & Jones (1927) \\
\hline \multirow{3}{*}{ Hyalopterus amygdali } & Lepisiota nigra & Mortazavi et al. (2015) \\
\hline & Monomorium mayri & Shiran et al. (2013) \\
\hline & Plagiolepis pallescens & Shiran et al. (2013) \\
\hline \multirow{5}{*}{ Hyalopterus pruni } & Lasius brunneus & Akyürek et al. (2016) \\
\hline & Lasius paralienus & Shiran et al. (2013) \\
\hline & Lepisiota bipartite & Shiran et al. (2013) \\
\hline & Pogonomyrmex occidentalis & Jones (1927) \\
\hline & Tapinoma simrothi & Stary (1969) \\
\hline \multirow{3}{*}{ Hysteroneura setariae } & Camponotus navigator & Idechiil et al. (2007) \\
\hline & Tapinoma melanocephalum & Idechiil et al. (2007) \\
\hline & Technomyrmex albipes & Idechiil et al. (2007) \\
\hline \multirow{4}{*}{ Protaphis middletonii } & Camponotus vicinus & Jones (1927) \\
\hline & Formica cinerea & Jones (1927) \\
\hline & Formica fusca & Jones (1927) \\
\hline & Myrmica incompleta & Jones (1927) \\
\hline Protaphis terricola & Lasius turcicus & Özdemir et al. (2008) \\
\hline Rhopalosiphum cerasifoliae & Lasius alienus & Favret et al. (2010) \\
\hline Rhopalosiphum maidis & Formica cinerea & Jones (1927) \\
\hline
\end{tabular}




\begin{tabular}{|c|c|c|}
\hline & Formica fusca & Jones (1927) \\
\hline & Lasius alienus & Barton and Ives (2014) \\
\hline & Lepisiota bipartite & Shiran et al. (2013) \\
\hline & Monomorium mayri & Shiran et al. (2013) \\
\hline & Monomorium qarahe & Shiran et al. (2013) \\
\hline & Myrmica incompleta & Jones (1927) \\
\hline & Polyrhachis lacteipennis & Shiran et al. (2013) \\
\hline & Prenolepis imparis & Barton and Ives (2014) \\
\hline & Tapinoma simrothi & Stary (1969) \\
\hline \multirow{35}{*}{ Rhopalosiphum nymphaeae } & Formica argentea & Jones (1927) \\
\hline & Formica aserva & Jones (1927) \\
\hline & Formica bradleyi & Jones (1927) \\
\hline & Formica coloradensis & Jones (1927) \\
\hline & Formica gnava & Jones (1927) \\
\hline & Formica incerta & Jones (1927) \\
\hline & Formica integroides & Jones (1927) \\
\hline & Formica lasioides & Jones (1927) \\
\hline & Formica lepida & Jones (1927) \\
\hline & Formica limata & Jones (1927) \\
\hline & Formica munda & Jones (1927) \\
\hline & Formica neoclara & Jones (1927) \\
\hline & Formica neogagates & Jones (1927) \\
\hline & Formica neorufibarbis & Jones (1927) \\
\hline & Formica obscuripes & Jones (1927) \\
\hline & Formica oreas & Jones (1927) \\
\hline & Formica puberula & Jones (1927) \\
\hline & Formica ravida & Jones (1927) \\
\hline & Formica rubicunda & Jones (1927) \\
\hline & Formica rufa & Jones (1927) \\
\hline & Formica sanguinea & Jones (1927) \\
\hline & Formica subaenescens & Jones (1927) \\
\hline & Formica subintegra & Jones (1927) \\
\hline & Formica subsericea & Jones (1927) \\
\hline & Formica ulkei & Jones (1927) \\
\hline & Lasius alienus & Jones (1927) \\
\hline & Lasius claviger & Jones (1927) \\
\hline & Lasius latipes & Jones (1927) \\
\hline & Lasius nearcticus & Jones (1927) \\
\hline & Lasius neoniger & Jones (1927) \\
\hline & Lasius pallitarsis & Jones (1927) \\
\hline & Leptothorax muscorum & Jones (1927) \\
\hline & Liometopum luctuosum & Jones (1927) \\
\hline & Liometopum occidentale & Jones (1927) \\
\hline & Monomorium minimum & Jones (1927) \\
\hline Rhopalosiphum padi & Formica aquilonia & Novgorodova (2005) \\
\hline
\end{tabular}




\begin{tabular}{|c|c|c|}
\hline & Formica polyctena & Novgorodova (2005) \\
\hline & Lepisiota bipartite & Shiran et al. (2013) \\
\hline & Tapinoma simrothi & Shiran et al. (2013) \\
\hline Sanbornia juniperi & Nylanderia fulva & Sharma and Buss (2013) \\
\hline \multirow{2}{*}{ Schizaphis gramina } & Formica aquilonia & Novgorodova (2005) \\
\hline & Formica polyctena & Novgorodova (2005) \\
\hline Schizaphis graminum & Camponotus herculeanus & Jones (1927) \\
\hline Schizaphis miscanthi & Formica cinereofusca & Akyildirim et al. (2014) \\
\hline Schizaphis nigerrima & Lepisiota nigra & Mortazavi et al. (2015) \\
\hline \multirow{3}{*}{ Schizaphis pyri } & Formica aquilonia & Novgorodova (2005) \\
\hline & Formica polyctena & Novgorodova (2005) \\
\hline & Lasius brunneus & Akyürek et al. (2016) \\
\hline \multicolumn{3}{|l|}{ Tribe Macrosiphini } \\
\hline \multirow{2}{*}{ Acyrthosiphon gossypii } & Crematogaster inermis & Mortazavi et al. (2015) \\
\hline & Lasius turcicus & Mortazavi et al. (2015) \\
\hline \multirow{5}{*}{ Acyrthosiphon pisum } & Crematogaster inermis & Mortazavi et al. (2015) \\
\hline & Formica cunicularia & Mortazavi et al. (2015) \\
\hline & Lasius turcicus & Akyildirim et al. (2014), Akyürek et al. (2016) \\
\hline & Lepisiota dolabellae & Mortazavi et al. (2015) \\
\hline & Plagiolepis pallescens & Mortazavi et al. (2015) \\
\hline Acyrthosiphon rubi & Formica cunicularia & Mortazavi et al. (2015) \\
\hline \multirow{2}{*}{ Anuraphis subterranea } & Formica argentea & Jones (1927) \\
\hline & Formica fusca & Jones (1927) \\
\hline $\begin{array}{l}\text { Aphthargelia } \\
\text { symphoricarpi }\end{array}$ & Formica cinerea & Addicott (1978) \\
\hline \multirow{2}{*}{ Artemisaphis artemisicola } & Formica fusca & Jones (1927) \\
\hline & Myrmica incompleta & Jones (1927) \\
\hline Aulacorthum solani & Formica subsericea & Favret et al. (2010) \\
\hline \multirow{6}{*}{ Bipersona torticauda } & Camponotus vicinus & Jones (1927) \\
\hline & Formica altipetens & Jones (1927) \\
\hline & Formica argentea & Jones (1927) \\
\hline & Formica cinerea & Jones (1927) \\
\hline & Formica fusca & Jones (1927) \\
\hline & Tapinoma sessile & Jones (1927) \\
\hline Brachcaudus helicrysi & Camponotus aethiops & Özdemir et al. (2008) \\
\hline \multirow{3}{*}{ Brachycaudus amygdalinus } & Lasius turcicus & Mortazavi et al. (2015) \\
\hline & Proformica pilosiscapa & Mortazavi et al. (2015) \\
\hline & Tapinoma erraticum & Mortazavi et al. (2015) \\
\hline \multirow{7}{*}{ Brachycaudus cardui } & Brachymyrmex patagonica & Lescano et al. (2015) \\
\hline & Camponotus aethiops & Özdemir et al. (2008), Akyürek et al. (2016) \\
\hline & Crematogaster sordidula & Özdemir et al. (2008), Mortazavi et al. (2015) \\
\hline & Dorymyrmex tener & Lescano et al. (2015) \\
\hline & Dorymyrmex wolffuegeli & Lescano et al. (2015) \\
\hline & Formica cinereofusca & Akyildirim et al. (2014) \\
\hline & Formica fusca & Jones (1927), Akyildirim et al. (2014) \\
\hline
\end{tabular}




\begin{tabular}{|c|c|c|}
\hline & Formica glauca & Özdemir et al. (2008) \\
\hline & Formica rufa & Jones (1927) \\
\hline & Lasius alienus & Akyürek et al. (2016) \\
\hline & Lasius brunneus & Akyürek et al. (2016) \\
\hline & Lasius emarginatus & Akyildirim et al. (2014) \\
\hline & Lasius niger & $\begin{array}{l}\text { Volkl et al. (1999), Müller and Godfray } \\
\text { (1999), Woodring et al. (2004) }\end{array}$ \\
\hline & Lasius paralienus & Özdemir et al. (2008) \\
\hline & Lasius turcicus & Akyildirim et al. (2014), Akyürek et al. (2016) \\
\hline & Myrmica incompleta & Jones (1927) \\
\hline & Myrmica ruginodis & $\begin{array}{l}\text { Müller and Godfray (1999), Akyildirim et al. } \\
\text { (2014) }\end{array}$ \\
\hline & Plagiolepis pygmaea & Akyürek et al. (2016) \\
\hline & Solenopsis richteri & Lescano et al. (2015) \\
\hline & Tapinoma sessile & Jones (1927) \\
\hline & Tapinoma simrothi & Stary (1969) \\
\hline & Tetramorium caespitum & Akyürek et al. (2016) \\
\hline & Tetramorium forte & Akyildirim et al. (2014) \\
\hline \multirow{7}{*}{ Brachycaudus helichrysi } & Aphaenogaster kurdica & Mortazavi et al. (2015) \\
\hline & Formica cinerea & Jones (1927) \\
\hline & Formica cinereofusca & Akyildirim et al. (2014) \\
\hline & Lasius turcicus & Akyürek et al. (2016) \\
\hline & Lepisiota nigra & Mortazavi et al. (2015) \\
\hline & Myrmica scabrinodis & Akyildirim et al. (2014) \\
\hline & Tapinoma simrothi & Shiran et al. (2013) \\
\hline Brachycaudus lychnidis & Formica cinereofusca & Akyildirim et al. (2014) \\
\hline \multirow{2}{*}{ Brachycaudus persicae } & Formica fusca & Jones (1927) \\
\hline & Myrmica scabrinodis & Jones (1927) \\
\hline Brachycaudus prunicola & Myrmica incompleta & Jones (1927) \\
\hline Brachycaudus schwartzi & Lasius emarginatus & Akyildirim et al. (2014) \\
\hline \multirow{8}{*}{$\begin{array}{l}\text { Brachycaudus } \\
\text { tragopogonis }\end{array}$} & Crematogaster inermis & Mortazavi et al. (2015) \\
\hline & Formica cunicularia & Mortazavi et al. (2015) \\
\hline & Lepisiota dolabellae & Mortazavi et al. (2015) \\
\hline & Lepisiota nigra & Mortazavi et al. (2015) \\
\hline & Plagiolepis pallescens & Mortazavi et al. (2015) \\
\hline & Tapinoma erraticum & Mortazavi et al. (2015) \\
\hline & Formica glauca & Özdemir et al. (2008) \\
\hline & Lasius alienus & Özdemir et al. (2008) \\
\hline \multirow{2}{*}{ Capitophorus elaeagni } & Crematogaster lineolata & Favret et al. (2010) \\
\hline & Prenolepis imparis & Favret et al. (2010) \\
\hline Capitophorus hippophaes & Lasius alienus & Özdemir et al. (2008) \\
\hline Capitophorus inulae & Tapinoma simrothi & Shiran et al. (2013) \\
\hline Ceruraphis viburnicola & Formica fusca & Jones (1927) \\
\hline Chaetosiphon tetrarhodum & Lasius turcicus & Akyildirim et al. (2014) \\
\hline Colorado absinthiella & Tapinoma simrothi & Shiran et al. (2013) \\
\hline
\end{tabular}




\begin{tabular}{|c|c|c|}
\hline \multirow{3}{*}{ Cryptomyzus ribis } & Formica argentea & Jones (1927) \\
\hline & Formica fusca & Jones (1927) \\
\hline & Myrmica scabrinodis & Jones (1927) \\
\hline Diuraphis calamagrostis & Formica cinerea & Jones (1927) \\
\hline Dysaphis devecta & Formica rufibarbis & Akyürek et al. (2016) \\
\hline Dysaphis foeniculus & Lasius alienus & Akyürek et al. (2016) \\
\hline \multirow{2}{*}{ Dysaphis plantaginea } & Lasius brunneus & Akyürek et al. (2016) \\
\hline & Lasius niger & Nagy et al. (2015) \\
\hline Dysaphis pyri & Lasius alienus & Akyildirim et al. (2014) \\
\hline \multirow{2}{*}{ Dysaphis sorbi } & Formica fusca & Jones (1927) \\
\hline & Tapinoma sessile & Jones (1927) \\
\hline Hyadaphis foeniculi & Lasius paralienus & Özdemir et al. (2008) \\
\hline Hyadaphis hofmanni & Camponotus piceus & Özdemir et al. (2008) \\
\hline Hyperomyzus lactucae & Formica argentea & Jones (1927) \\
\hline Hyperomyzus nabali & Formica subsericea & Favret et al. (2010) \\
\hline Macrosiphon rosae & Lepisiota nigra & Mortazavi et al. (2015) \\
\hline \multirow{2}{*}{ Macrosiphoniella pulvera } & Formica cunicularia & Novgorodova (2005) \\
\hline & Formica polyctena & Novgorodova (2005) \\
\hline Macrosiphoniella sanborni & Tetramorium caespitum & Akyildirim et al. (2014) \\
\hline \multirow{3}{*}{ Macrosiphum chrysothamni } & Camponotus vicinus & Jones (1927) \\
\hline & Formica fusca & Jones (1927) \\
\hline & Formica montana & Jones (1927) \\
\hline \multirow{2}{*}{ Macrosiphum euphorbiae } & Cataglyphis aenescens & Mortazavi et al. (2015) \\
\hline & Formica argentea & Jones (1927) \\
\hline Macrosiphum rosae & Formica cunicularia & Akyürek et al. (2016) \\
\hline Macrosiphum valerianae & Camponotus vicinus & Jones (1927) \\
\hline Metopeurum fuscoviride & Lasius niger & $\begin{array}{l}\text { Volkl et al. (1999), Fischer et al. (2002), } \\
\text { Woodring et al. (2004) }\end{array}$ \\
\hline $\begin{array}{l}\text { Microsiphoniella } \\
\text { artemisiae }\end{array}$ & Formica sp. & Jones (1927) \\
\hline \multirow{2}{*}{ Myzaphis rosarum } & Formica argentea & Jones (1927) \\
\hline & Formica fusca & Jones (1927) \\
\hline \multirow{5}{*}{ Myzus cerasi } & Lasius alienus & Akyürek et al. (2016) \\
\hline & Lasius emarginatus & Akyildirim et al. (2014) \\
\hline & Lasius turcicus & Akyildirim et al. (2014), Akyürek et al. (2016) \\
\hline & Proformica kobachidzei & Akyildirim et al. (2014) \\
\hline & Formica fusca & Jones (1927) \\
\hline \multirow{4}{*}{ Myzus lythri } & Formica cinereofusca & Akyildirim et al. (2014) \\
\hline & Lasius emarginatus & Akyildirim et al. (2014) \\
\hline & Lasius turcicus & Akyildirim et al. (2014) \\
\hline & Tetramorium caespitum & Akyürek et al. (2016) \\
\hline Myzus oenotherae & Formica fusca & Jones (1927) \\
\hline Myzus ornatus & Formica rufibarbis & Akyürek et al. (2016) \\
\hline Myzus padellus & Formica rufibarbis & Akyürek et al. (2016) \\
\hline Myzus persicae & Camponotus compressus & Kataria and Kumar (2013) \\
\hline
\end{tabular}




\begin{tabular}{|c|c|c|}
\hline & Cataglyphis cinnamomeus & Shiran et al. (2013) \\
\hline & Crematogaster antaris & Shiran et al. (2013) \\
\hline & Formica argentea & Jones (1927) \\
\hline & Lasius paralienus & Shiran et al. (2013) \\
\hline & Lepisiota bipartite & Shiran et al. (2013) \\
\hline & Linepithema humile & Powell and Silverman (2010) \\
\hline & Monomorium mayri & Shiran et al. (2013) \\
\hline & Pheidole pallidula & Shiran et al. (2013) \\
\hline & Pogonomyrmex occidentalis & Jones (1927) \\
\hline & Solenopsis invicta & Zhou (2014), Zhou (2012) \\
\hline & Tapinoma melanocephalum & Zhou et al. (2015) \\
\hline & Tapinoma sessile & Powell and Silverman (2010) \\
\hline & Tapinoma simrothi & Shiran et al. (2013) \\
\hline Myzus varians & Lasius turcicus & Akyürek et al. (2016) \\
\hline Nasonovia houghtonensis & Formica fusca & Jones (1927) \\
\hline \multirow{4}{*}{ Nearctaphis bakeri } & Formica cinereofusca & Akyildirim et al. (2014) \\
\hline & Formica rufa & Jones (1927) \\
\hline & Prenolepis imparis & Favret et al. (2010) \\
\hline & Tapinoma simrothi & Shiran et al. (2013) \\
\hline Neomyzus circumflexum & Formica subsericea & Favret et al. (2010) \\
\hline \multirow{3}{*}{ Obtusicauda frigidae } & Camponotus herculeanus & Jones (1927) \\
\hline & Formica efiniventris & Jones (1927) \\
\hline & Formica fusca & Jones (1927) \\
\hline Ovatus crataegarius & Formica sp. & Jones (1927) \\
\hline \multirow{2}{*}{$\begin{array}{l}\text { Pleotrichophorus } \\
\text { glandulosus }\end{array}$} & Formica montana & Jones (1927) \\
\hline & Formica cinerea & Addicott (1978) \\
\hline Pleotrichophorus utensis & Formica obscuripes & Billick et al. (2007) \\
\hline $\begin{array}{c}\text { Pseudoepameibaphis } \\
\text { tridentatae }\end{array}$ & Formica montana & Jones (1927) \\
\hline \multirow{2}{*}{ Pterocomma bicolor } & Formica fusca & Jones (1927) \\
\hline & Myrmica incompleta & Jones (1927) \\
\hline Pterocomma pilosum & Lasius fuliginosus & Molnar et al. (2000) \\
\hline \multirow{3}{*}{ Pterocomma populeum } & Camponotus herculeanus & Jones (1927) \\
\hline & Lasius brunneus & Akyürek et al. (2016) \\
\hline & Lasius turcicus & Akyürek et al. (2016) \\
\hline \multirow{2}{*}{ Pterocomma rufipes } & Cremastogaster scutellaris & Akyürek et al. (2016) \\
\hline & Lasius fuliginosus & Molnar et al. (2000) \\
\hline \multirow{2}{*}{ Pterocomma salicis } & Formica fusca & Jones (1927) \\
\hline & Myrmica incompleta & Jones (1927) \\
\hline Pterocomma smithiae & Formica argentea & Jones (1927) \\
\hline Sitobion fragariae & Lasius brunneus & Akyürek et al. (2016) \\
\hline \multirow{2}{*}{ Staegeriella necopinata } & Camponotus piceus & Özdemir et al. (2008) \\
\hline & Plagiolepis vindobonensis & Özdemir et al. (2008) \\
\hline \multirow{2}{*}{$\begin{array}{l}\text { Uroleucon ambrosiae } \\
\text { ambrosiae }\end{array}$} & Formica argentea & Jones (1927) \\
\hline & Formica canadensis & Jones (1927) \\
\hline
\end{tabular}




\begin{tabular}{|c|c|c|}
\hline & Formica cinerea & Jones (1927) \\
\hline & Formica fusca & Jones (1927) \\
\hline & Myrmica incompleta & Jones (1927) \\
\hline Uroleucon Dactynotus & Monomorium gracillimum & Stary (1969) \\
\hline \multirow{3}{*}{ Uroleucon erigeronense } & Formica argentea & Jones (1927) \\
\hline & Formica cinerea & Jones (1927) \\
\hline & Formica fusca & Jones (1927) \\
\hline Uroleucon escalantii & Formica obscuripes & Billick et al. (2007) \\
\hline Uroleucon kashmiricum & Lasius brunneus & Akyürek et al. (2016) \\
\hline Uroleucon kikioense & Formica fusca & Akyildirim et al. (2014) \\
\hline $\begin{array}{c}\text { Uroleucon } \\
\text { nigrotuberculatum }\end{array}$ & Formica japonica & Ando and Ohgushi (2008) \\
\hline Uroleucon pieloui & Formica subsericea & Favret et al. (2010) \\
\hline Uroleucon solidaginis & Formica argentea & Jones (1927) \\
\hline Uroleucon sonchellum & Prenolepis imparis & Favret et al. (2010) \\
\hline Uroleucon sonchi & Tetramorium forte & Akyürek et al. (2016) \\
\hline Uroleucon taraxaci & Formica argentea & Jones (1927) \\
\hline Wahlgreniella nervata & Myrmica incompleta & Jones (1927) \\
\hline \multicolumn{3}{|l|}{ Subfamily Calaphidinae } \\
\hline \multicolumn{3}{|l|}{ Tribe Calaphidini } \\
\hline \multirow{4}{*}{ Callipterinella tuberculata } & Camponotus saxatilis & Novgorodova (2005) \\
\hline & Formica aquilonia & Novgorodova (2005) \\
\hline & Formica polyctena & Novgorodova (2005) \\
\hline & Lasius niger & Novgorodova (2005) \\
\hline \multirow{2}{*}{ Neobetulaphis pusilla } & Lasius alienus & Akyildirim et al. (2014) \\
\hline & Lasius turcicus & Akyildirim et al. (2014) \\
\hline \multirow{7}{*}{ Symydobius oblongus } & Camponotus saxatilis & Novgorodova (2005) \\
\hline & Formica aquilonia & Novgorodova (2005) \\
\hline & Formica cinereofusca & Akyildirim et al. (2014) \\
\hline & Formica polyctena & Novgorodova (2005) \\
\hline & Formica pratensis & Novgorodova (2005) \\
\hline & Lasius fuliginosus & Novgorodova (2005) \\
\hline & Lasius niger & Novgorodova (2005) \\
\hline \multicolumn{3}{|l|}{ Tribe Panaphidini } \\
\hline Chromaphis obiensis & Lasius niger & Novgorodova (2005) \\
\hline \multirow{2}{*}{ Myzocallis discolor } & Formica argentea & Jones (1927) \\
\hline & Formica fusca & Jones (1927) \\
\hline Myzocallis kuricola & Lasius niger & Sakata (1995) \\
\hline Shivaphis celti & Nylanderia fulva & Sharma and Buss (2013) \\
\hline Pterocallis albidus & Formica cinereofusca & Akyildirim et al. (2014) \\
\hline Pterocallis alni & Formica cinereofusca & Akyildirim et al. (2014) \\
\hline Tinocallis platani & Plagiolepis pallescens & Shiran et al. (2013) \\
\hline Tuberculatus quercicola & Formica yessensis & $\begin{array}{c}\text { Yao and Akimoto (2001), Yao and Akimoto } \\
\text { (2009), Yao and Akimoto (2009) }\end{array}$ \\
\hline Tuberculatus maximus & Crematogaster subdentata & Mortazavi et al. (2015) \\
\hline
\end{tabular}




\begin{tabular}{|c|c|c|}
\hline $\begin{array}{c}\text { Subfamily } \\
\text { Chaitophorinae }\end{array}$ & & \\
\hline \multicolumn{3}{|l|}{ Tribe Chaitophorini } \\
\hline Chaitophorous populialbae & Polyrhachis simplex & Degen et al. (1986) \\
\hline \multirow{2}{*}{ Chaitophorus albitorosus } & Formica aquilonia & Novgorodova (2005) \\
\hline & Formica polyctena & Novgorodova (2005) \\
\hline \multirow{5}{*}{ Chaitophorus albus } & Camponotus saxatilis & Novgorodova (2005) \\
\hline & Formica aquilonia & Novgorodova (2005) \\
\hline & Formica cunicularia & Novgorodova (2005) \\
\hline & Formica polyctena & Novgorodova (2005) \\
\hline & Lasius niger & Novgorodova (2005) \\
\hline \multirow{3}{*}{ Chaitophorus euphraticus } & Lepisiota bipartite & Shiran et al. (2013) \\
\hline & Monomorium destructor & Shiran et al. (2013) \\
\hline & Monomorium mayri & Shiran et al. (2013) \\
\hline $\begin{array}{l}\text { Chaitophorus } \\
\text { hillerislambersi }\end{array}$ & Lasius turcicus & Mortazavi et al. (2015) \\
\hline \multirow{5}{*}{ Chaitophorus israeliticus } & Crematogaster inermis & Mortazavi et al. (2015) \\
\hline & Crematogaster schmidti & Mortazavi et al. (2015) \\
\hline & Lepisiota nigra & Mortazavi et al. (2015) \\
\hline & Messor orientalis & Mortazavi et al. (2015) \\
\hline & Tapinoma erraticum & Mortazavi et al. (2015) \\
\hline Chaitophorus kapuri & Lasius alienus & Akyildirim et al. (2014) \\
\hline \multirow{3}{*}{ Chaitophorus longisetosus } & Formica cinereofusca & Akyildirim et al. (2014) \\
\hline & Lasius emarginatus & Akyildirim et al. (2014) \\
\hline & Lasius paralienus & Akyildirim et al. (2014) \\
\hline \multirow{2}{*}{$\begin{array}{l}\text { Chaitophorus } \\
\text { macrostachyae }\end{array}$} & Formica fusca & Jones (1927) \\
\hline & Myrmica incompleta & Jones (1927) \\
\hline $\begin{array}{l}\text { Chaitophorus } \\
\text { melanosiphon }\end{array}$ & Crematogaster scutellaris & Akyürek et al. (2016) \\
\hline Chaitophorus nassonowi & Formica pratensis & Novgorodova (2005) \\
\hline \multirow[t]{2}{*}{ Chaitophorus nigrae } & $\begin{array}{c}\text { Camponotus } \\
\text { novaeboracensis }\end{array}$ & Jones (1927) \\
\hline & Formica argentea & Jones (1927) \\
\hline \multirow{10}{*}{ Chaitophorus populeti } & Camponotus saxatilis & Novgorodova (2005) \\
\hline & Formica aquilonia & Novgorodova (2005) \\
\hline & Formica cunicularia & Novgorodova (2005) \\
\hline & Formica fusca & Novgorodova (2005) \\
\hline & Formica polyctena & Novgorodova (2005) \\
\hline & Formica pratensis & Novgorodova (2005) \\
\hline & Formica sanguinea & Akyürek et al. (2016) \\
\hline & Lasius fuliginosus & Novgorodova (2005) \\
\hline & Lasius niger & $\begin{array}{l}\text { Fischer and Shingleton (2001), Novgorodova } \\
\qquad(2005)\end{array}$ \\
\hline & Lasius paralienus & Shiran et al. (2013) \\
\hline \multirow{2}{*}{ Chaitophorus populialbae } & Camponotus saxatilis & Novgorodova (2005) \\
\hline & Formica aquilonia & Novgorodova (2005) \\
\hline
\end{tabular}




\begin{tabular}{|c|c|c|}
\hline & Formica cunicularia & Novgorodova (2005) \\
\hline & Formica fusca & Novgorodova (2005) \\
\hline & Formica polyctena & Novgorodova (2005) \\
\hline & Lasius niger & $\begin{array}{l}\text { Fischer and Shingleton (2001), Novgorodova } \\
\text { (2005) }\end{array}$ \\
\hline & Lasius paralienus & Shiran et al. (2013) \\
\hline \multirow{9}{*}{ Chaitophorus populicola } & Camponotus herculeanus & Jones (1927) \\
\hline & Camponotus pennsylvanicus & Jones (1927) \\
\hline & Formica argentea & Jones (1927) \\
\hline & Formica dakotensis & Jones (1927) \\
\hline & Formica montana & Jones (1927) \\
\hline & Formica propinqua & Wimp and Whitham (2001) \\
\hline & Linepithema humile & Mondor and Addicott (2007) \\
\hline & Myrmica incompleta & Jones (1927) \\
\hline & Tapinoma sessile & Jones (1927) \\
\hline \multirow{8}{*}{ Chaitophorus populifolii } & Camponotus herculeanus & Jones (1927) \\
\hline & Camponotus vicinus & Jones (1927) \\
\hline & Formica argentea & Jones (1927) \\
\hline & Formica aterrima & Jones (1927) \\
\hline & Formica canadensis & Jones (1927) \\
\hline & Formica fusca & Jones (1927) \\
\hline & Formica rufa & Jones (1927) \\
\hline & Tapinoma sessile & Jones (1927) \\
\hline Chaitophorus remaudierei & Lasius paralienus & Shiran et al. (2013) \\
\hline \multirow{3}{*}{ Chaitophorus salicti } & Formica aquilonia & Novgorodova (2005) \\
\hline & Formica polyctena & Novgorodova (2005) \\
\hline & Lasius turcicus & Akyildirim et al. (2014) \\
\hline \multirow{3}{*}{ Chaitophorus saliniger } & Camponotus japonicus & Hembry et al. (2006) \\
\hline & Formica japonica & Hembry et al. (2006) \\
\hline & Lasius japonicus & Hembry et al. (2006) \\
\hline \multirow{2}{*}{ Chaitophorus tremulae } & Camponotus saxatilis & Novgorodova (2005) \\
\hline & Lasius niger & Novgorodova (2005) \\
\hline \multirow{3}{*}{ Chaitophorus truncates } & Crematogaster antaris & Shiran et al. (2013) \\
\hline & Lasius paralienus & Shiran et al. (2013) \\
\hline & Lepisiota bipartite & Shiran et al. (2013) \\
\hline \multirow{6}{*}{ Chaitophorus viminalis } & Camponotus vicinus & Jones (1927) \\
\hline & Formica argentea & Jones (1927) \\
\hline & Formica fusca & Jones (1927) \\
\hline & Myrmecina graminicola & Jones (1927) \\
\hline & Myrmica whymperi & Jones (1927) \\
\hline & Tapinoma sessile & Jones (1927) \\
\hline \multirow{2}{*}{ Chaitophorus viminicola } & Crematogaster lineolata & Favret et al. (2010) \\
\hline & Nylanderia fulva & Sharma and Buss (2013) \\
\hline Chaitophorus vitellinae & Lasius fuliginosus & Molnar et al. (2000) \\
\hline Periphyllus aceris & Formica cinereofusca & Akyildirim et al. (2014) \\
\hline
\end{tabular}




\begin{tabular}{|c|c|c|}
\hline & Lasius brunneus & Akyürek et al. (2016) \\
\hline & Lasius emarginatus & Akyildirim et al. (2014) \\
\hline \multirow{3}{*}{ Periphyllus bulgaricus } & Crematogaster subdentata & Mortazavi et al. (2015) \\
\hline & Lasius alienus & Mortazavi et al. (2015) \\
\hline & Lepisiota nigra & Mortazavi et al. (2015) \\
\hline \multirow{3}{*}{ Periphyllus negundinis } & Dorymyrmex pyramicus & Jones (1927) \\
\hline & Formica argentea & Jones (1927) \\
\hline & Formica fusca & Jones (1927) \\
\hline \multicolumn{3}{|l|}{ Tribe Siphini } \\
\hline \multirow{3}{*}{ Sipha maydis } & Formica cinereofusca & Akyildirim et al. (2014) \\
\hline & Formica cunicularia & Akyürek et al. (2016) \\
\hline & Lasius alienus & Akyürek et al. (2016) \\
\hline \multicolumn{3}{|l|}{$\begin{array}{c}\text { Subfamily } \\
\text { Drepanosiphinae }\end{array}$} \\
\hline \multirow{3}{*}{ Drepanaphis acerifoliae } & Formica argentea & Jones (1927) \\
\hline & Formica canadensis & Jones (1927) \\
\hline & Myrmica scabrinodis & Jones (1927) \\
\hline \multirow{2}{*}{ Drepanaphis nigricans } & Camponotus subbarbatus & Favret et al. (2010) \\
\hline & Myrmica punctiventris & Favret et al. (2010) \\
\hline \multirow{2}{*}{ Drepanosiphum braggii } & Formica canadensis & Jones (1927) \\
\hline & Formica fusca & Jones (1927) \\
\hline \multicolumn{3}{|l|}{ Subfamily Eriosomatinae } \\
\hline \multicolumn{3}{|l|}{ Tribe Eriosomatini } \\
\hline Eriosoma americanum & Formica cinerea & Jones (1927) \\
\hline Eriosoma crataegi & Myrmica incompleta & Jones (1927) \\
\hline Tetraneura sp. & Technomyrmex albipes & Idechiil et al. (2007) \\
\hline \multicolumn{3}{|l|}{ Tribe Fordini } \\
\hline \multirow{3}{*}{ Forda marginata } & Lasius americanus & Cockerell (1903) \\
\hline & Lasius claviger & Cockerell (1903) \\
\hline & Lasius flavus & Cockerell (1903) \\
\hline Geoica sp. & Formica fusca & Jones (1927) \\
\hline Paracletus cimiciformis & Tetramorium semilaeve & Salazar et al. (2015) \\
\hline \multicolumn{3}{|l|}{ Tribe Pemphigini } \\
\hline Pachypappa sacculi & Formica sp. & Jones (1927) \\
\hline Pemphigus lasii & Lasius americanus & Cockerell (1903), Palmer (1952) \\
\hline $\begin{array}{c}\text { Pemphigus } \\
\text { populiramulorum }\end{array}$ & Pheidole pilifera & Jones (1927) \\
\hline \multirow{8}{*}{ Pentalonia nigronervosa } & Anoplolepis gracilipes & Idechiil et al. (2007) \\
\hline & Camponotus reticulatus & Idechiil et al. (2007) \\
\hline & Cardiocondyla emeryi & Idechiil et al. (2007) \\
\hline & Cardiocondyla wroughtonii & Idechiil et al. (2007) \\
\hline & Paratrechina bourbonica & Idechiil et al. (2007) \\
\hline & Tapinoma melanocephalum & Idechiil et al. (2007) \\
\hline & Technomyrmex albipes & Idechiil et al. (2007) \\
\hline & Tetramorium bicarinatum & Idechiil et al. (2007) \\
\hline
\end{tabular}




\begin{tabular}{|c|c|c|}
\hline \multirow{2}{*}{ Prociphilus fraxinifolii } & Formica argentea & Jones (1927) \\
\hline & Formica cinerea & Jones (1927) \\
\hline \multirow{3}{*}{ Thecabius populimonilis } & Camponotus sansabeanus & Jones (1927) \\
\hline & Camponotus vicinus & Jones (1927) \\
\hline & Pheidole bicarinata & Jones (1927) \\
\hline \multicolumn{3}{|l|}{ Subfamily Lachninae } \\
\hline \multicolumn{3}{|l|}{ Tribe Eulachnini } \\
\hline Cinara acutirostris & Formica cinereofusca & Akyildirim et al. (2014) \\
\hline \multirow{6}{*}{ Cinara apini } & Camponotus herculeanus & Jones (1927) \\
\hline & Camponotus maculatus & Jones (1927) \\
\hline & Formica argentea & Jones (1927) \\
\hline & Formica comata & Jones (1927) \\
\hline & Formica dakotensis & Jones (1927) \\
\hline & Myrmica rubra & Jones (1927) \\
\hline \multirow{3}{*}{ Cinara atlantica } & Formica subsericea & Favret et al. (2010) \\
\hline & Lasius alienus & Favret et al. (2010) \\
\hline & Tapinoma sessile & Favret et al. (2010) \\
\hline \multirow{6}{*}{ Cinara boerneri } & Formica aquilonia & Novgorodova (2005) \\
\hline & Formica cunicularia & Novgorodova (2005) \\
\hline & Formica fusca & Novgorodova (2005) \\
\hline & Formica polyctena & Novgorodova (2005) \\
\hline & Formica pratensis & Novgorodova (2005) \\
\hline & Lasius fuliginosus & Novgorodova (2005) \\
\hline \multirow{2}{*}{ Cinara brevispinosa } & Formica fusca & Jones (1927) \\
\hline & Myrmica scabrinodis & Jones (1927) \\
\hline \multirow{2}{*}{ Cinara cedri } & Lasius brunneus & Akyürek et al. (2016) \\
\hline & Tapinoma simrothi & Shiran et al. (2013) \\
\hline \multirow{2}{*}{ Cinara coloradensis } & Camponotus pennsylvanicus & Jones (1927) \\
\hline & Formica cinerea & Jones (1927) \\
\hline \multirow{3}{*}{ Cinara cupressi } & Formica cinerea & Jones (1927) \\
\hline & Lasius brunneus & Akyürek et al. (2016) \\
\hline & Lasius turcicus & Akyürek et al. (2016) \\
\hline \multirow{3}{*}{ Cinara edulis } & Camponotus vicinus & Jones (1927) \\
\hline & Crematogaster lineolata & Jones (1927) \\
\hline & Formica fusca & Jones (1927) \\
\hline Cinara flexilis & Camponotus pennsylvanicus & Jones (1927) \\
\hline \multirow{2}{*}{ Cinara hottesi } & Camponotus herculeanus & Jones (1927) \\
\hline & Formica cinerea & Jones (1927) \\
\hline Cinara indica & Formica rufibarbis & Akyürek et al. (2016) \\
\hline Cinara juniperivora & Nylanderia fulva & Sharma and Buss (2013) \\
\hline \multirow{5}{*}{ Cinara laricis } & Formica aquilonia & Novgorodova (2005) \\
\hline & Formica fusca & Novgorodova (2005) \\
\hline & Formica polyctena & Novgorodova (2005) \\
\hline & Formica pratensis & Novgorodova (2005) \\
\hline & Lasius fuliginosus & Novgorodova (2005) \\
\hline
\end{tabular}




\begin{tabular}{|c|c|c|}
\hline Cinara maritimae & Formica cinereofusca & Akyildirim et al. (2014) \\
\hline Cinara medispinosa & Formica montana & Jones (1927) \\
\hline Cinara murrayanae & Crematogaster lineolata & Jones (1927) \\
\hline Cinara occidentalis & Formica rufibarbis & Akyürek et al. (2016) \\
\hline \multirow{2}{*}{ Cinara oregonensis } & Camponotus vicinus & Jones (1927) \\
\hline & Myrmica incompleta & Jones (1927) \\
\hline Cinara palaestinensis & Lasius paralienus & Shiran et al. (2013) \\
\hline \multirow{5}{*}{ Cinara palaestinesis } & Cataglyphis emeryi & LATIBARI et al. (2016) \\
\hline & Formica cunicularia & LATIBARI et al. (2016) \\
\hline & Lasius alienus & LATIBARI et al. (2016) \\
\hline & Lasius paralienus & LATIBARI et al. (2016) \\
\hline & Pheidole pallidula & LATIBARI et al. (2016) \\
\hline \multirow[t]{2}{*}{ Cinara piceae } & Formica aquilonia & $\begin{array}{c}\text { Novgorodova (2005), Gibb and Johansson } \\
\text { (2010) }\end{array}$ \\
\hline & Formica polyctena & Novgorodova (2005) \\
\hline Cinara piceicola & Formica aquilonia & Gibb and Johansson (2010) \\
\hline \multirow{4}{*}{ Cinara pilicornis } & Formica cinereofusca & Akyildirim et al. (2014) \\
\hline & Formica fusca & Akyildirim et al. (2014) \\
\hline & Lasius turcicus & Akyildirim et al. (2014) \\
\hline & Tetramorium forte & Akyildirim et al. (2014) \\
\hline \multirow{5}{*}{ Cinara pinea } & Formica aquilonia & Novgorodova (2005) \\
\hline & Formica fusca & Novgorodova (2005) \\
\hline & Formica polyctena & Novgorodova (2005) \\
\hline & Formica pratensis & Novgorodova (2005) \\
\hline & Lasius fuliginosus & Novgorodova (2005) \\
\hline Cinara pineti & Tapinoma sessile & Jones (1927) \\
\hline \multirow{18}{*}{ Cinara pini } & Camponotus saxatilis & Novgorodova (2005) \\
\hline & Cardiocondyla shalbergi & LATIBARI et al. (2016) \\
\hline & Cataglyphis aenescens & LATIBARI et al. (2016) \\
\hline & Cataglyphis emeryi & LATIBARI et al. (2016) \\
\hline & Cataglyphis nodus & LATIBARI et al. (2016) \\
\hline & Crematogaster subdentata & LATIBARI et al. (2016) \\
\hline & Formica aquilonia & Novgorodova (2005) \\
\hline & Formica cunicularia & Novgorodova (2005) \\
\hline & Formica fusca & Novgorodova (2005) \\
\hline & Formica polyctena & Novgorodova (2005) \\
\hline & Formica pratensis & Novgorodova (2005) \\
\hline & Lasius alienus & LATIBARI et al. (2016) \\
\hline & Lasius emarginatus & Akyildirim et al. (2014) \\
\hline & Lasius niger & Novgorodova (2005) \\
\hline & Lasius paralienus & LATIBARI et al. (2016) \\
\hline & Lasius turcicus & Akyildirim et al. (2014) \\
\hline & Lepisiota nigra & LATIBARI et al. (2016) \\
\hline & Tapinoma erraticum & LATIBARI et al. (2016) \\
\hline Cinara pinihabitans & Camponotus saxatilis & Novgorodova (2005) \\
\hline
\end{tabular}




\begin{tabular}{|c|c|c|}
\hline Cinara pinivora & Formica subsericea & Favret et al. (2010) \\
\hline \multirow{4}{*}{ Cinara ponderosae } & Camponotus vicinus & Jones (1927) \\
\hline & Crematogaster laeviuscula & Jones (1927) \\
\hline & Formica argentea & Jones (1927) \\
\hline & Tapinoma sessile & Jones (1927) \\
\hline Cinara pruinosa & Formica cinereofusca & Akyildirim et al. (2014) \\
\hline \multirow{6}{*}{ Cinara pseudotsugae } & Camponotus herculeanus & Jones (1927) \\
\hline & Camponotus vicinus & Jones (1927) \\
\hline & Formica canadensis & Jones (1927) \\
\hline & Formica cinerea & Jones (1927) \\
\hline & Formica fusca & Jones (1927) \\
\hline & Formica montana & Jones (1927) \\
\hline \multirow{4}{*}{ Cinara schwarzii } & $\begin{array}{c}\text { Camponotus } \\
\text { novaeboracensis }\end{array}$ & Jones (1927) \\
\hline & Camponotus vicinus & Jones (1927) \\
\hline & Crematogaster lineolata & Jones (1927) \\
\hline & Formica comata & Jones (1927) \\
\hline Cinara sibiricae & Camponotus vicinus & Jones (1927) \\
\hline \multirow{2}{*}{ Cinara splendens } & Camponotus herculeanus & Jones (1927) \\
\hline & Formica comata & Jones (1927) \\
\hline Cinara strobi & Camponotus pennsylvanicus & Favret et al. (2010) \\
\hline \multirow{2}{*}{ Cinara tujafilina } & Lasius brunneus & Akyürek et al. (2016) \\
\hline & Lasius turcicus & Akyürek et al. (2016) \\
\hline Cinara vandykei & Camponotus herculeanus & Jones (1927) \\
\hline $\begin{array}{c}\text { Eulachnus } \\
\text { tuberculostemmatus }\end{array}$ & Camponotus turkestanicus & Mortazavi et al. (2015) \\
\hline $\begin{array}{c}\text { Eulachnus } \\
\text { tuberculostommata }\end{array}$ & Tapinoma simrothi & Shiran et al. (2013) \\
\hline Schizolachnus pineti & Formica cinereofusca & Akyildirim et al. (2014) \\
\hline \multicolumn{3}{|l|}{ Tribe Lachnini } \\
\hline Lachnus ater & Crematogaster lineolata & Jones (1927) \\
\hline Lachnus glaber & Formica argentea & Jones (1927) \\
\hline \multirow{5}{*}{ Lachnus roboris } & Camponotus aethiops & Akyürek et al. (2016) \\
\hline & Lasius brunneus & Akyürek et al. (2016) \\
\hline & Lasius grandis & Paris and Espadaler (2009) \\
\hline & Lasius neglectus & Paris and Espadaler (2009) \\
\hline & Proformica kobachidzei & Akyildirim et al. (2014) \\
\hline \multirow[t]{2}{*}{ Lachnus solitarius } & $\begin{array}{c}\text { Camponotus } \\
\text { novaeboracensis }\end{array}$ & Jones (1927) \\
\hline & Camponotus vicinus & Jones (1927) \\
\hline \multirow{2}{*}{ Lachnus tropicalis } & Lasius fuliginosus & Zhang et al. (2015) \\
\hline & Lasius niger & Sakata (1995) \\
\hline \multirow{4}{*}{ Pterochloroides persicae } & Cataglyphis aenescens & Mortazavi et al. (2015) \\
\hline & Cataglyphis nodus & Mortazavi et al. (2015) \\
\hline & Lasius paralienus & Shiran et al. (2013) \\
\hline & Lasius turcicus & Akyildirim et al. (2014) \\
\hline
\end{tabular}




\begin{tabular}{|c|c|c|}
\hline & Lepisiota bipartite & Shiran et al. (2013) \\
\hline & Lepisiota frauenfeldi & Mortazavi et al. (2015) \\
\hline & Lepisiota nigra & Mortazavi et al. (2015) \\
\hline & Tapinoma simrothi & Stary (1969) \\
\hline Stomaphis hirukawai & Lasius productus & Matsuura and Yashiro (2006) \\
\hline Stomaphis japonica & Lasius fuliginosus & Zhang et al. (2015) \\
\hline Stomaphis quercus & Lasius fuliginosus & Novgorodova (2005) \\
\hline Stomaphis yanonis & Lasius fuji & Endo and Itino (2013) \\
\hline \multicolumn{3}{|l|}{ Tribe Tramini } \\
\hline Trama troglodytes & Lasius niger & Woodring et al. (2004) \\
\hline \multicolumn{3}{|l|}{ Tribe Tuberolachnini } \\
\hline \multirow{3}{*}{ Tuberolachnus salignus } & Formica canadensis & Jones (1927) \\
\hline & Formica fusca & Jones (1927) \\
\hline & Formica rufibarbis & Akyürek et al. (2016) \\
\hline \multicolumn{3}{|l|}{ Subfamily Thelaxinae } \\
\hline \multicolumn{3}{|l|}{ Tribe Thelaxini } \\
\hline \multirow{3}{*}{ Glyphina betulae } & Camponotus saxatilis & Novgorodova (2005) \\
\hline & Formica aquilonia & Novgorodova (2005) \\
\hline & Formica polyctena & Novgorodova (2005) \\
\hline \multirow{2}{*}{ Thelaxes californica } & Crematogaster schmidti & Akyildirim et al. (2014) \\
\hline & Lasius emarginatus & Akyildirim et al. (2014) \\
\hline \multirow{2}{*}{ Thelaxes suberi } & Camponotus aethiops & Akyürek et al. (2016) \\
\hline & Lasius brunneus & Akyürek et al. (2016) \\
\hline \multirow{6}{*}{ Thelaxes suberis } & Camponotus gestroi & Stary (1969) \\
\hline & Camponotus kurdistanicus & Stary (1969) \\
\hline & Crematogaster auberti & Stary (1969) \\
\hline & Crematogaster sordidula & Stary (1969) \\
\hline & Pheidole pallidula & Stary (1969) \\
\hline & Tapinoma simrothi & Stary (1969) \\
\hline \multicolumn{3}{|l|}{$\begin{array}{c}\text { Subfamily } \\
\text { Saltusaphidinae }\end{array}$} \\
\hline \multicolumn{3}{|l|}{ Tribe Thripsaphidini } \\
\hline \multirow{2}{*}{ Callaphis juglandis } & Crematogaster inermis & Mortazavi et al. (2015) \\
\hline & Plagiolepis pallescens & Mortazavi et al. (2015) \\
\hline
\end{tabular}

Table A2. Species interactions of aphid-associated ants

\begin{tabular}{c|c|c}
\hline Ants & Associated aphids & Reference \\
\hline Subfamily Dolichoderinae & & \\
\hline Tribe Leptomyrmecini & & Jones (1927) \\
\hline \multirow{2}{*}{ Dorymyrmex pyramicus } & Aphis lugentis & Jones (1927) \\
\cline { 2 - 3 } & Periphyllus negundinis & Lescano et al. (2015) \\
\hline Dorymyrmex tener & Brachycaudus cardui & Lescano et al. (2015) \\
\hline Dorymyrmex wolffuegeli & Brachycaudus cardui & Idechiil et al. (2007) \\
\hline Iridomyrmex anceps & Aphis gossypii &
\end{tabular}




\begin{tabular}{|c|c|c|}
\hline Iridomyrmex humilis & Aphis nerii & Bristow (1991) \\
\hline Iridomyrmex hartmeyeri & Aphis clerodendri & Carver et al. (2003) \\
\hline \multirow{6}{*}{ Linepithema humile } & Aphis coreopsidis & Altfeld and Stiling (2009) \\
\hline & Aphis gossypii & $\begin{array}{l}\text { Powell and Silverman (2010), Tena et al. } \\
\text { (2013), LeVan and Holway (2015) }\end{array}$ \\
\hline & Aphis nerii & Pringle et al. (2014) \\
\hline & Aphis spiraecola & Tena et al. (2013) \\
\hline & Chaitophorus populicola & Mondor and Addicott (2007) \\
\hline & Myzus persicae & Powell and Silverman (2010) \\
\hline \multicolumn{3}{|l|}{ Tribe Tapinomini } \\
\hline Liometopum luctuosum & Rhopalosiphum nymphaeae & Jones (1927) \\
\hline Liometopum occidentale & Rhopalosiphum nymphaeae & Jones (1927) \\
\hline \multirow{9}{*}{ Tapinoma erraticum } & Aphis craccivora & Mortazavi et al. (2015) \\
\hline & Aphis fabae & Mortazavi et al. (2015) \\
\hline & Aphis gossypii & Mortazavi et al. (2015) \\
\hline & Aphis pomi & Mortazavi et al. (2015) \\
\hline & Aphis salicariae & Mortazavi et al. (2015) \\
\hline & Brachycaudus amygdalinus & Mortazavi et al. (2015) \\
\hline & Brachycaudus tragopogonis & Mortazavi et al. (2015) \\
\hline & Chaitophorus israeliticus & Mortazavi et al. (2015) \\
\hline & Cinara pini & LATIBARI et al. (2016) \\
\hline Tapinoma indicum & Aphis gossypii & Lokeshwari et al. (2015) \\
\hline \multirow{4}{*}{ Tapinoma melanocephalum } & Aphis gossypii & $\begin{array}{c}\text { Idechiil et al. (2007), Lokeshwari et al. } \\
\text { (2015) }\end{array}$ \\
\hline & Hysteroneura setariae & Idechiil et al. (2007) \\
\hline & Myzus persicae & Zhou et al. (2015) \\
\hline & Pentalonia nigronervosa & Idechiil et al. (2007) \\
\hline \multirow{16}{*}{ Tapinoma sessile } & Aphis asclepiadis & Addicott (1979a), Jones (1927) \\
\hline & Aphis gossypii & Powell and Silverman (2010) \\
\hline & Aphis helianthi & Mooney et al. (2015) \\
\hline & Aphis lugentis & Favret et al. (2010) \\
\hline & Aphis salicariae & Addicott (1979a), Mooney et al. (2015) \\
\hline & Aphis varians & Addicott (1979a), Mooney et al. (2015) \\
\hline & Bipersona torticauda & Jones (1927) \\
\hline & Brachycaudus cardui & Jones (1927) \\
\hline & Chaitophorus populicola & Jones (1927) \\
\hline & Chaitophorus populifolii & Jones (1927) \\
\hline & Chaitophorus viminalis & Jones (1927) \\
\hline & Cinara atlantica & Favret et al. (2010) \\
\hline & Cinara pineti & Jones (1927) \\
\hline & Cinara ponderosae & Jones (1927) \\
\hline & Dysaphis sorbi & Jones (1927) \\
\hline & Myzus persicae & Powell and Silverman (2010) \\
\hline \multirow{2}{*}{ Tapinoma simrothi } & Aphis craccivora & Stary (1969), Shiran et al. (2013) \\
\hline & Aphis davletshinae & Shiran et al. (2013) \\
\hline
\end{tabular}




\begin{tabular}{|c|c|c|}
\hline & Aphis fabae & Stary (1969), Shiran et al. (2013) \\
\hline & Aphis frangulae & Shiran et al. (2013) \\
\hline & Aphis gossypii & Stary (1969) \\
\hline & Aphis nasturtii & Shiran et al. (2013) \\
\hline & Aphis nerii & Shiran et al. (2013) \\
\hline & Aphis pomi & Stary (1969) \\
\hline & Aphis punicae & Stary (1969) \\
\hline & Aphis rumicis & Shiran et al. (2013) \\
\hline & Aphis umbrella & Shiran et al. (2013) \\
\hline & Brachycaudus cardui & Stary (1969) \\
\hline & Brachycaudus helichrysi & Shiran et al. (2013) \\
\hline & Brachyonguis harmalae & Shiran et al. (2013) \\
\hline & $\begin{array}{l}\text { Brachyonguis } \\
\text { tamaricophilus }\end{array}$ & Shiran et al. (2013) \\
\hline & Capitophorus inulae & Shiran et al. (2013) \\
\hline & Cinara cedri & Shiran et al. (2013) \\
\hline & Colorado absinthiella & Shiran et al. (2013) \\
\hline & $\begin{array}{c}\text { Eulachnus } \\
\text { tuberculostommata }\end{array}$ & Shiran et al. (2013) \\
\hline & Hyalopterus pruni & Stary (1969) \\
\hline & Myzus persicae & Shiran et al. (2013) \\
\hline & Nearctaphis bakeri & Shiran et al. (2013) \\
\hline & Pterochloroides persicae & Stary (1969) \\
\hline & Rhopalosiphum maidis & Stary (1969) \\
\hline & Rhopalosiphum padi & Shiran et al. (2013) \\
\hline & Thelaxes suberis & Stary (1969) \\
\hline \multirow{4}{*}{ Technomyrmex albipes } & Aphis clerodendri & Carver et al. (2003) \\
\hline & Aphis gossypii & $\begin{array}{l}\text { Idechiil et al. (2007), Lokeshwari et al. } \\
\text { (2015) }\end{array}$ \\
\hline & Hysteroneura setariae & Idechiil et al. (2007) \\
\hline & Pentalonia nigronervosa & Idechiil et al. (2007) \\
\hline \multicolumn{3}{|l|}{ Subfamily Formicinae } \\
\hline \multicolumn{3}{|l|}{ Tribe Camponotini } \\
\hline \multirow{7}{*}{ Camponotus aethiops } & Aphis brotericola & Özdemir et al. (2008) \\
\hline & Aphis fabae & Akyürek et al. (2016) \\
\hline & Aphis salviae & Özdemir et al. (2008) \\
\hline & Brachcaudus helicrysi & Özdemir et al. (2008) \\
\hline & Brachycaudus cardui & Özdemir et al. (2008), Akyürek et al. (2016) \\
\hline & Lachnus roboris & Akyürek et al. (2016) \\
\hline & Thelaxes suberi & Akyürek et al. (2016) \\
\hline \multirow{4}{*}{ Camponotus compressus } & Aphis craccivora & $\begin{array}{l}\text { Kataria and Kumar (2013), Rakhshan and } \\
\text { Ahmad (2015) }\end{array}$ \\
\hline & Aphis fabae & Kataria and Kumar (2013) \\
\hline & Aphis gossypii & $\begin{array}{l}\text { Verghese and Tandon (1987), Kataria and } \\
\text { Kumar (2013), Lokeshwari et al. (2015) }\end{array}$ \\
\hline & Aphis nerii & Kataria and Kumar (2013) \\
\hline
\end{tabular}




\begin{tabular}{|c|c|c|}
\hline & Myzus persicae & Kataria and Kumar (2013) \\
\hline Camponotus gestroi & Thelaxes suberis & Stary (1969) \\
\hline \multirow{14}{*}{ Camponotus herculeanus } & Aphis lugentis & Jones (1927) \\
\hline & Aphis salicariae & Addicott (1979a) \\
\hline & Aphis valerianae & Jones (1927) \\
\hline & Aphis varians & Addicott (1979a) \\
\hline & Chaitophorus populicola & Jones (1927) \\
\hline & Chaitophorus populifolii & Jones (1927) \\
\hline & Cinara apini & Jones (1927) \\
\hline & Cinara hottesi & Jones (1927) \\
\hline & Cinara pseudotsugae & Jones (1927) \\
\hline & Cinara splendens & Jones (1927) \\
\hline & Cinara vandykei & Jones (1927) \\
\hline & Obtusicauda frigidae & Jones (1927) \\
\hline & Pterocomma populeum & Jones (1927) \\
\hline & Schizaphis graminum & Jones (1927) \\
\hline Camponotus invidus & Aphis craccivora & Rakhshan and Ahmad (2015) \\
\hline Camponotus japonicus & Chaitophorus saliniger & Hembry et al. (2006) \\
\hline \multirow{2}{*}{ Camponotus kurdistanicus } & Thelaxes suberis & Stary (1969) \\
\hline & Aphis craccivora & Stary (1969) \\
\hline Camponotus lateralis & Aphis spiraecola & Akyürek et al. (2016) \\
\hline Camponotus libanicus & Aphis craccivora & Shiran et al. (2013) \\
\hline Camponotus maculatus & Cinara apini & Jones (1927) \\
\hline Camponotus modoc & Aphis lugentis & Jones (1927) \\
\hline Camponotus navigator & Hysteroneura setariae & Idechiil et al. (2007) \\
\hline \multirow{3}{*}{$\begin{array}{c}\text { Camponotus } \\
\text { novaeboracensis }\end{array}$} & Chaitophorus nigrae & Jones (1927) \\
\hline & Cinara schwarzii & Jones (1927) \\
\hline & Lachnus solitarius & Jones (1927) \\
\hline \multirow{2}{*}{ Camponotus oasium } & Aphis craccivora & Shiran et al. (2013) \\
\hline & Aphis frangulae & Shiran et al. (2013) \\
\hline Camponotus parius & Aphis gossypii & Lokeshwari et al. (2015) \\
\hline \multirow{4}{*}{ Camponotus pennsylvanicus } & Chaitophorus populicola & Jones (1927) \\
\hline & Cinara coloradensis & Jones (1927) \\
\hline & Cinara flexilis & Jones (1927) \\
\hline & Cinara strobi & Favret et al. (2010) \\
\hline \multirow{8}{*}{ Camponotus piceus } & Aphis brotericola & Özdemir et al. (2008) \\
\hline & Aphis chloris & Özdemir et al. (2008) \\
\hline & Aphis craccivora & Özdemir et al. (2008) \\
\hline & Aphis euphorbiae & Özdemir et al. (2008) \\
\hline & Aphis fabae & Özdemir et al. (2008) \\
\hline & Aphis salviae & Özdemir et al. (2008) \\
\hline & Hyadaphis hofmanni & Özdemir et al. (2008) \\
\hline & Staegeriella necopinata & Özdemir et al. (2008) \\
\hline \multirow{2}{*}{ Camponotus reticulatus } & Aphis gossypii & Idechiil et al. (2007) \\
\hline & Pentalonia nigronervosa & Idechiil et al. (2007) \\
\hline
\end{tabular}




\begin{tabular}{|c|c|c|}
\hline Camponotus sansabeanus & Thecabius populimonilis & Jones (1927) \\
\hline \multirow{11}{*}{ Camponotus saxatilis } & Aphis brohmeri & Novgorodova (2005) \\
\hline & Aphis fabae & Novgorodova (2005) \\
\hline & Callipterinella tuberculata & Novgorodova (2005) \\
\hline & Chaitophorus albus & Novgorodova (2005) \\
\hline & Chaitophorus populeti & Novgorodova (2005) \\
\hline & Chaitophorus populialbae & Novgorodova (2005) \\
\hline & Chaitophorus tremulae & Novgorodova (2005) \\
\hline & Cinara pini & Novgorodova (2005) \\
\hline & Cinara pinihabitans & Novgorodova (2005) \\
\hline & Glyphina betulae & Novgorodova (2005) \\
\hline & Symydobius oblongus & Novgorodova (2005) \\
\hline Camponotus subbarbatus & Drepanaphis nigricans & Favret et al. (2010) \\
\hline Camponotus turkestanicus & $\begin{array}{c}\text { Eulachnus } \\
\text { tuberculostemmatus }\end{array}$ & Mortazavi et al. (2015) \\
\hline \multirow{17}{*}{ Camponotus vicinus } & Aphis asclepiadis & Jones (1927) \\
\hline & Aphis valerianae & Jones (1927) \\
\hline & Bipersona torticauda & Jones (1927) \\
\hline & Chaitophorus populifolii & Jones (1927) \\
\hline & Chaitophorus viminalis & Jones (1927) \\
\hline & Cinara edulis & Jones (1927) \\
\hline & Cinara oregonensis & Jones (1927) \\
\hline & Cinara ponderosae & Jones (1927) \\
\hline & Cinara pseudotsugae & Jones (1927) \\
\hline & Cinara schwarzii & Jones (1927) \\
\hline & Cinara sibiricae & Jones (1927) \\
\hline & Lachnus solitarius & Jones (1927) \\
\hline & Macrosiphum chrysothamni & Jones (1927) \\
\hline & Macrosiphum valerianae & Jones (1927) \\
\hline & Protaphis middletonii & Jones (1927) \\
\hline & Thecabius populimonilis & Jones (1927) \\
\hline & Aphis clerodendri & Carver et al. (2003) \\
\hline Polyrhachis hauxwelli & Aphis craccivora & Rakhshan and Ahmad (2015) \\
\hline \multirow{3}{*}{ Polyrhachis lacteipennis } & Aphis craccivora & Shiran et al. (2013) \\
\hline & Aphis frangulae & Shiran et al. (2013) \\
\hline & Rhopalosiphum maidis & Shiran et al. (2013) \\
\hline Polyrhachis simplex & Chaitophorous populialbae & Degen et al. (1986) \\
\hline \multirow{6}{*}{ Prenolepis imparis } & Aphis cornifoliae & Favret et al. (2010) \\
\hline & Aphis fabae & Favret et al. (2010) \\
\hline & Capitophorus elaeagni & Favret et al. (2010) \\
\hline & Nearctaphis bakeri & Favret et al. (2010) \\
\hline & Rhopalosiphum maidis & Barton and Ives (2014) \\
\hline & Uroleucon sonchellum & Favret et al. (2010) \\
\hline \multicolumn{3}{|l|}{ Tribe Formicini } \\
\hline Cataglyphis aenescens & Aphis craccivora & Özdemir et al. (2008) \\
\hline
\end{tabular}




\begin{tabular}{|c|c|c|}
\hline & Cinara pini & LATIBARI et al. (2016) \\
\hline & Macrosiphum euphorbiae & Mortazavi et al. (2015) \\
\hline & Pterochloroides persicae & Mortazavi et al. (2015) \\
\hline Cataglyphis cinnamomeus & Myzus persicae & Shiran et al. (2013) \\
\hline \multirow{2}{*}{ Cataglyphis emeryi } & Cinara palaestinesis & LATIBARI et al. (2016) \\
\hline & Cinara pini & LATIBARI et al. (2016) \\
\hline Cataglyphis livida & Subcryptosiphon sp. & Stary (1969) \\
\hline Cataglyphis lividus & Aphis fabae & Shiran et al. (2013) \\
\hline \multirow{5}{*}{ Cataglyphis nodus } & Aphis frangulae & Shiran et al. (2013) \\
\hline & Aphis gossypii & Stary (1969) \\
\hline & Chaitophorus sp. & Stary (1969) \\
\hline & Cinara pini & LATIBARI et al. (2016) \\
\hline & Pterochloroides persicae & Mortazavi et al. (2015) \\
\hline Cataglyphis protuberata & Lachnus sp. & Stary (1969) \\
\hline Formica altipetens & Bipersona torticauda & Jones (1927) \\
\hline \multirow{25}{*}{ Formica aquilonia } & Aphis brohmeri & Novgorodova (2005) \\
\hline & Aphis craccivora & Novgorodova (2005) \\
\hline & Aphis evonymi & Novgorodova (2005) \\
\hline & Aphis fabae & Novgorodova (2005) \\
\hline & Aphis jacobaeae & Novgorodova (2005) \\
\hline & Aphis pomi & Novgorodova (2005) \\
\hline & Aphis subnitidae & Novgorodova (2005) \\
\hline & Aphis viburni & Novgorodova (2005) \\
\hline & Callipterinella tuberculata & Novgorodova (2005) \\
\hline & Chaitophorus albitorosus & Novgorodova (2005) \\
\hline & Chaitophorus albus & Novgorodova (2005) \\
\hline & Chaitophorus populeti & Novgorodova (2005) \\
\hline & Chaitophorus populialbae & Novgorodova (2005) \\
\hline & Chaitophorus salicti & Novgorodova (2005) \\
\hline & Cinara boerneri & Novgorodova (2005) \\
\hline & Cinara laricis & Novgorodova (2005) \\
\hline & Cinara piceae & $\begin{array}{c}\text { Novgorodova (2005), Gibb and Johansson } \\
(2010)\end{array}$ \\
\hline & Cinara piceicola & Gibb and Johansson (2010) \\
\hline & Cinara pinea & Novgorodova (2005) \\
\hline & Cinara pini & Novgorodova (2005) \\
\hline & Glyphina betulae & Novgorodova (2005) \\
\hline & Rhopalosiphum padi & Novgorodova (2005) \\
\hline & Schizaphis gramina & Novgorodova (2005) \\
\hline & Schizaphis pyri & Novgorodova (2005) \\
\hline & Symydobius oblongus & Novgorodova (2005) \\
\hline \multirow{4}{*}{ Formica argentea } & Anuraphis subterranea & Jones (1927) \\
\hline & Aphis aiddletonii & Jones (1927) \\
\hline & Aphis asclepiadis & Jones (1927) \\
\hline & Aphis gossypii & Jones (1927) \\
\hline
\end{tabular}




\begin{tabular}{|c|c|c|}
\hline & Aphis lugentis & Jones (1927) \\
\hline & Aphis medicaginis & Jones (1927) \\
\hline & Aphis sambucifolii & Jones (1927) \\
\hline & Aphis ulmariae & Jones (1927) \\
\hline & Bipersona torticauda & Jones (1927) \\
\hline & Braggia eriogoni & Jones (1927) \\
\hline & Chaitophorus nigrae & Jones (1927) \\
\hline & Chaitophorus populicola & Jones (1927) \\
\hline & Chaitophorus populifolii & Jones (1927) \\
\hline & Chaitophorus viminalis & Jones (1927) \\
\hline & Cinara apini & Jones (1927) \\
\hline & Cinara ponderosae & Jones (1927) \\
\hline & Cryptomyzus ribis & Jones (1927) \\
\hline & Drepanaphis acerifoliae & Jones (1927) \\
\hline & Hyperomyzus lactucae & Jones (1927) \\
\hline & Lachnus glaber & Jones (1927) \\
\hline & Macrosiphum euphorbiae & Jones (1927) \\
\hline & Myzaphis rosarum & Jones (1927) \\
\hline & Myzocallis discolor & Jones (1927) \\
\hline & Myzus persicae & Jones (1927) \\
\hline & Periphyllus negundinis & Jones (1927) \\
\hline & Prociphilus fraxinifolii & Jones (1927) \\
\hline & Pterocomma smithiae & Jones (1927) \\
\hline & Rhopalosiphum nymphaeae & Jones (1927) \\
\hline & Uroleucon ambrosiae & Jones (1927) \\
\hline & Uroleucon erigeronense & Jones (1927) \\
\hline & Uroleucon solidaginis & Jones (1927) \\
\hline & Uroleucon taraxaci & Jones (1927) \\
\hline \multirow{4}{*}{ Formica aserva } & Aphis asclepiadis & Addicott (1979a) \\
\hline & Aphis salicariae & Addicott (1979a) \\
\hline & Aphis varians & Addicott (1979a) \\
\hline & Rhopalosiphum nymphaeae & Jones (1927) \\
\hline Formica aterrima & Chaitophorus populifolii & Jones (1927) \\
\hline Formica bradleyi & Rhopalosiphum nymphaeae & Jones (1927) \\
\hline \multirow{11}{*}{ Formica canadensis } & Aphis asclepiadis & Jones (1927) \\
\hline & Aphis lugentis & Jones (1927) \\
\hline & Aphis medicaginis & Jones (1927) \\
\hline & Aphis pomi & Jones (1927) \\
\hline & Aphis valerianae & Jones (1927) \\
\hline & Chaitophorus populifolii & Jones (1927) \\
\hline & Cinara pseudotsugae & Jones (1927) \\
\hline & Drepanaphis acerifoliae & Jones (1927) \\
\hline & Drepanosiphum braggii & Jones (1927) \\
\hline & Tuberolachnus salignus & Jones (1927) \\
\hline & Uroleucon ambrosiae & Jones (1927) \\
\hline
\end{tabular}




\begin{tabular}{|c|c|c|}
\hline \multirow{21}{*}{ Formica cinerea } & Aphis asclepiadis & Jones (1927) \\
\hline & Aphis forbesi & Jones (1927) \\
\hline & Aphis gossypii & Jones (1927) \\
\hline & Aphis medicaginis & Jones (1927) \\
\hline & Aphis pomi & Jones (1927) \\
\hline & Aphis varians & Addicott (1978), Breton and Addicott (1992) \\
\hline & Aphthargelia symphoricarpi & Addicott (1978) \\
\hline & Bipersona torticauda & Jones (1927) \\
\hline & Brachycaudus helichrysi & Jones (1927) \\
\hline & Cinara coloradensis & Jones (1927) \\
\hline & Cinara cupressi & Jones (1927) \\
\hline & Cinara hottesi & Jones (1927) \\
\hline & Cinara pseudotsugae & Jones (1927) \\
\hline & Diuraphis calamagrostis & Jones (1927) \\
\hline & Eriosoma americanum & Jones (1927) \\
\hline & $\begin{array}{c}\text { Pleotrichophorus } \\
\text { quadritrichus }\end{array}$ & Addicott (1978) \\
\hline & Prociphilus fraxinifolii & Jones (1927) \\
\hline & Protaphis middletonii & Jones (1927) \\
\hline & Rhopalosiphum maidis & Jones (1927) \\
\hline & Uroleucon ambrosiae & Jones (1927) \\
\hline & Uroleucon erigeronense & Jones (1927) \\
\hline \multirow{3}{*}{ Formica cinerea lepida } & Aphis asclepiadis & Addicott (1979a) \\
\hline & Aphis salicariae & Addicott (1979a) \\
\hline & Aphis varians & Addicott (1979a) \\
\hline \multirow{20}{*}{ Formica cinereofusca } & Aphis craccivora & Akyildirim et al. (2014) \\
\hline & Aphis fabae & Akyildirim et al. (2014) \\
\hline & Aphis farinosa & Akyildirim et al. (2014) \\
\hline & Aphis gossypii & Akyildirim et al. (2014) \\
\hline & Aphis nasturtii & Akyildirim et al. (2014) \\
\hline & Aphis sambuci & Akyildirim et al. (2014) \\
\hline & Aphis serpylli & Akyildirim et al. (2014) \\
\hline & Aphis spiraecola & Akyildirim et al. (2014) \\
\hline & Aphis verbasci & Akyildirim et al. (2014) \\
\hline & Brachycaudus cardui & Akyildirim et al. (2014) \\
\hline & Brachycaudus helichrysi & Akyildirim et al. (2014) \\
\hline & Brachycaudus lychnidis & Akyildirim et al. (2014) \\
\hline & Brachyunguis tamaricis & Akyildirim et al. (2014) \\
\hline & Chaitophorus longisetosus & Akyildirim et al. (2014) \\
\hline & Cinara acutirostris & Akyildirim et al. (2014) \\
\hline & Cinara maritimae & Akyildirim et al. (2014) \\
\hline & Cinara pilicornis & Akyildirim et al. (2014) \\
\hline & Cinara pruinosa & Akyildirim et al. (2014) \\
\hline & Myzus lythri & Akyildirim et al. (2014) \\
\hline & Nearctaphis bakeri & Akyildirim et al. (2014) \\
\hline
\end{tabular}




\begin{tabular}{|c|c|c|}
\hline & Periphyllus aceris & Akyildirim et al. (2014) \\
\hline & Pterocallis albidus & Akyildirim et al. (2014) \\
\hline & Pterocallis alni & Akyildirim et al. (2014) \\
\hline & Schizaphis miscanthi & Akyildirim et al. (2014) \\
\hline & Schizolachnus pineti & Akyildirim et al. (2014) \\
\hline & Sipha maydis & Akyildirim et al. (2014) \\
\hline & Symydobius oblongus & Akyildirim et al. (2014) \\
\hline & Toxoptera aurantii & Akyildirim et al. (2014) \\
\hline Formica coloradensis & Rhopalosiphum nymphaeae & Jones (1927) \\
\hline \multirow{3}{*}{ Formica comata } & Cinara apini & Jones (1927) \\
\hline & Cinara schwarzii & Jones (1927) \\
\hline & Cinara splendens & Jones (1927) \\
\hline \multirow{7}{*}{ Formica cunicularia } & Aphis pomi & Novgorodova (2005) \\
\hline & Chaitophorus albus & Novgorodova (2005) \\
\hline & Chaitophorus populeti & Novgorodova (2005) \\
\hline & Chaitophorus populialbae & Novgorodova (2005) \\
\hline & Cinara boerneri & Novgorodova (2005) \\
\hline & Cinara pini & Novgorodova (2005) \\
\hline & Macrosiphoniella pulvera & Novgorodova (2005) \\
\hline \multirow{13}{*}{ Formica cunicularia } & Acyrthosiphon pisum & Mortazavi et al. (2015) \\
\hline & Acyrthosiphon rubi & Mortazavi et al. (2015) \\
\hline & Aphis acetosae & Mortazavi et al. (2015) \\
\hline & Aphis brotericola & Akyürek et al. (2016) \\
\hline & Aphis craccivora & $\begin{array}{l}\text { Özdemir et al. (2008), Mortazavi et al. } \\
\text { (2015) }\end{array}$ \\
\hline & Aphis fabae & Akyürek et al. (2016) \\
\hline & Aphis nasturtii & Akyürek et al. (2016) \\
\hline & Aphis solanella & Akyürek et al. (2016) \\
\hline & Aphis spiraecola & Akyürek et al. (2016) \\
\hline & Brachycaudus tragopogonis & Mortazavi et al. (2015) \\
\hline & Cinara palaestinesis & LATIBARI et al. (2016) \\
\hline & Macrosiphum rosae & Akyürek et al. (2016) \\
\hline & Sipha maydis & Akyürek et al. (2016) \\
\hline \multirow{2}{*}{ Formica dakotensis } & Chaitophorus populicola & Jones (1927) \\
\hline & Cinara apini & Jones (1927) \\
\hline \multirow{2}{*}{ Formica efiniventris } & Aphis lugentis & Jones (1927) \\
\hline & Obtusicauda frigidae & Jones (1927) \\
\hline \multirow{8}{*}{ Formica fusca } & Anuraphis subterranea & Jones (1927) \\
\hline & Aphis asclepiadis & Jones (1927), Addicott (1979a) \\
\hline & Aphis craccivora & Akyürek et al. (2016) \\
\hline & Aphis esulae & Akyürek et al. (2016) \\
\hline & Aphis fabae & Akyildirim et al. (2014) \\
\hline & Aphis farinosa & Jones (1927) \\
\hline & Aphis gossypii & Jones (1927) \\
\hline & Aphis helianthi & Mooney et al. (2015) \\
\hline
\end{tabular}




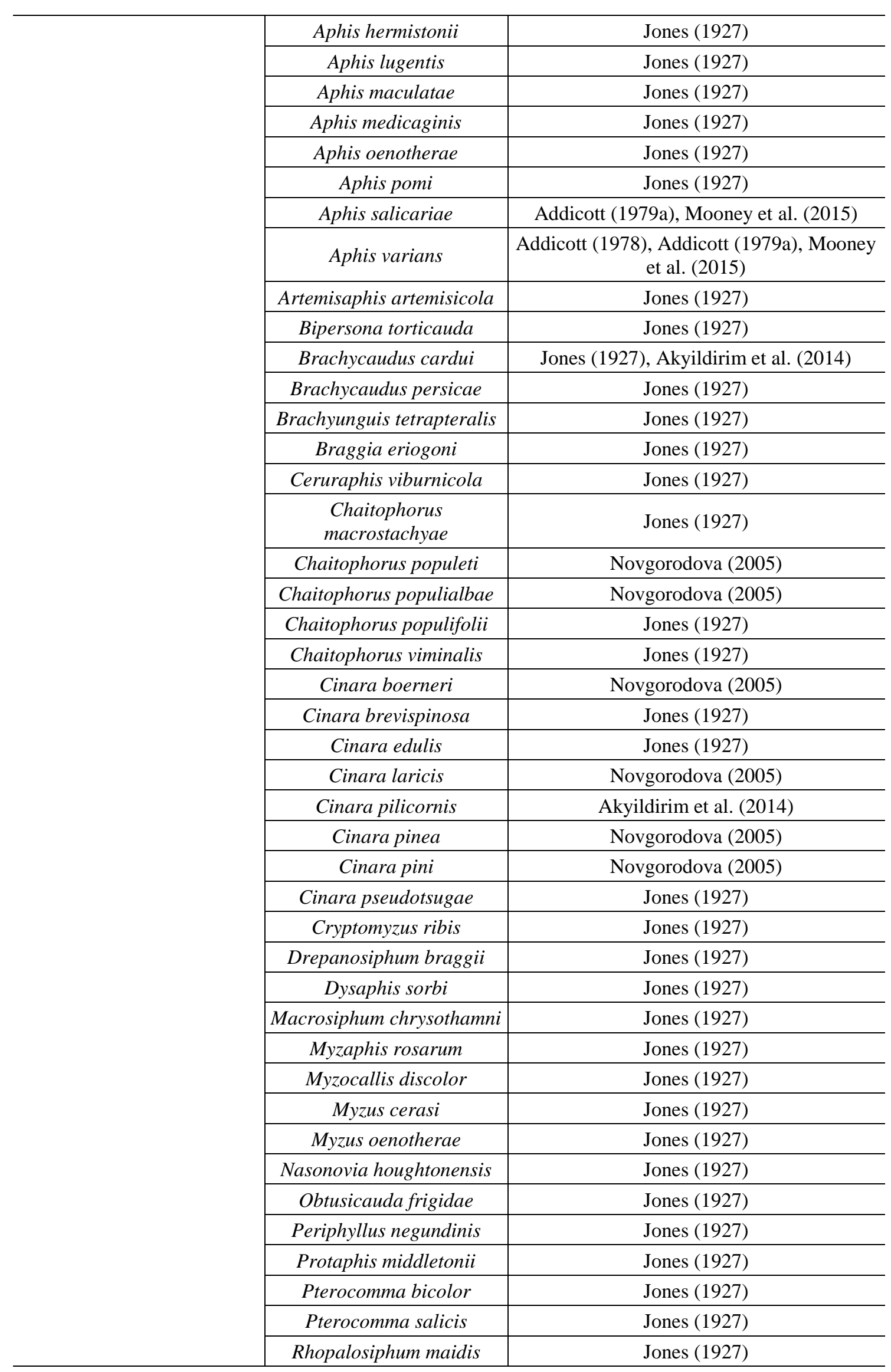




\begin{tabular}{|c|c|c|}
\hline & Tuberolachnus salignus & Jones (1927) \\
\hline & Uroleucon ambrosiae & Jones (1927) \\
\hline & Uroleucon erigeronense & Jones (1927) \\
\hline & Uroleucon kikioense & Akyildirim et al. (2014) \\
\hline Formica gagates & Aphis farinosa & Akyildirim et al. (2014) \\
\hline \multirow{5}{*}{ Formica glauca } & Aphis craccivora & Özdemir et al. (2008) \\
\hline & Aphis fabae & Özdemir et al. (2008) \\
\hline & Aphis galliiscabri & Özdemir et al. (2008) \\
\hline & $\begin{array}{c}\text { Brachycaudus (Appelia) } \\
\text { tragopogonis }\end{array}$ & Özdemir et al. (2008) \\
\hline & Brachycaudus cardui & Özdemir et al. (2008) \\
\hline Formica gnava & Rhopalosiphum nymphaeae & Jones (1927) \\
\hline Formica incerta & Rhopalosiphum nymphaeae & Jones (1927) \\
\hline \multirow{5}{*}{ Formica integroides } & Aphis farinosa & Addicott (1978) \\
\hline & Rhopalosiphum nymphaeae & Jones (1927) \\
\hline & Aphis asclepiadis & Addicott (1979a) \\
\hline & Aphis salicariae & Addicott (1979a) \\
\hline & Aphis varians & Addicott (1978), Addicott (1979a) \\
\hline \multirow[b]{2}{*}{ Formica japonica } & Chaitophorus saliniger & Hembry et al. (2006) \\
\hline & $\begin{array}{c}\text { Uroleucon } \\
\text { nigrotuberculatum }\end{array}$ & Ando and Ohgushi (2008) \\
\hline \multirow{4}{*}{ Formica lasioides } & Aphis asclepiadis & Addicott (1979a) \\
\hline & Aphis salicariae & Addicott (1979a) \\
\hline & Aphis varians & Addicott (1978), Addicott (1979a) \\
\hline & Rhopalosiphum nymphaeae & Jones (1927) \\
\hline \multirow{2}{*}{ Formica lepida } & Aphis asclepiadis & Jones (1927) \\
\hline & Rhopalosiphum nymphaeae & Jones (1927) \\
\hline Formica limata & Rhopalosiphum nymphaeae & Jones (1927) \\
\hline Formica lugubris & Cinara pini & Sudd (1983) \\
\hline \multirow{8}{*}{ Formica montana } & Aphis asclepiadis & Jones (1927) \\
\hline & Aphis oregonensis & Jones (1927) \\
\hline & Chaitophorus populicola & Jones (1927) \\
\hline & Cinara medispinosa & Jones (1927) \\
\hline & Cinara pseudotsugae & Jones (1927) \\
\hline & Macrosiphum chrysothamni & Jones (1927) \\
\hline & $\begin{array}{l}\text { Pleotrichophorus } \\
\text { glandulosus }\end{array}$ & Jones (1927) \\
\hline & $\begin{array}{c}\text { Pseudoepameibaphis } \\
\text { tridentatae }\end{array}$ & Jones (1927) \\
\hline Formica munda & Rhopalosiphum nymphaeae & Jones (1927) \\
\hline Formica neoclara & Rhopalosiphum nymphaeae & Jones (1927) \\
\hline Formica neogagates & Rhopalosiphum nymphaeae & Jones (1927) \\
\hline Formica neorufibarbis & Rhopalosiphum nymphaeae & Jones (1927) \\
\hline \multirow{3}{*}{ Formica neorufibarbis gelida } & Aphis asclepiadis & Addicott (1979a) \\
\hline & Aphis salicariae & Addicott (1979a) \\
\hline & Aphis varians & Addicott (1979a) \\
\hline
\end{tabular}




\begin{tabular}{|c|c|c|}
\hline \multirow{6}{*}{ Formica obscuripes } & Aphis asclepiadis & Addicott (1979a) \\
\hline & Aphis salicariae & Addicott (1979a) \\
\hline & Aphis varians & Addicott (1978), Addicott (1979a) \\
\hline & Pleotrichophorus utensis & Billick et al. (2007) \\
\hline & Rhopalosiphum nymphaeae & Jones (1927) \\
\hline & Uroleucon escalantii & Billick et al. (2007) \\
\hline \multirow{2}{*}{ Formica opaciventris } & Aphis asclepiadis & Jones (1927) \\
\hline & Aphis medicaginis & Jones (1927) \\
\hline Formica oreas & Rhopalosiphum nymphaeae & Jones (1927) \\
\hline \multirow[t]{2}{*}{ Formica podzolica } & Aphis asclepiadis & $\begin{array}{c}\text { Mooney et al. (2008), Nielsen et al. (2009), } \\
\text { Abdala-Roberts et al. (2012) }\end{array}$ \\
\hline & Aphis valerianae & Petry et al. (2012) \\
\hline \multirow{24}{*}{ Formica polyctena } & Aphis brohmeri & Novgorodova (2005) \\
\hline & Aphis craccivora & Novgorodova (2005) \\
\hline & Aphis evonymi & Novgorodova (2005) \\
\hline & Aphis fabae & Novgorodova (2005) \\
\hline & Aphis jacobaeae & Novgorodova (2005) \\
\hline & Aphis pomi & Novgorodova (2005) \\
\hline & Aphis subnitidae & Novgorodova (2005) \\
\hline & Callipterinella tuberculata & Novgorodova (2005) \\
\hline & Chaitophorus albitorosus & Novgorodova (2005) \\
\hline & Chaitophorus albus & Novgorodova (2005) \\
\hline & Chaitophorus populeti & Novgorodova (2005) \\
\hline & Chaitophorus populialbae & Novgorodova (2005) \\
\hline & Chaitophorus salicti & Novgorodova (2005) \\
\hline & Cinara boerneri & Novgorodova (2005) \\
\hline & Cinara laricis & Novgorodova (2005) \\
\hline & Cinara piceae & Novgorodova (2005) \\
\hline & Cinara pinea & Novgorodova (2005) \\
\hline & Cinara pini & Novgorodova (2005) \\
\hline & Glyphina betulae & Novgorodova (2005) \\
\hline & Macrosiphoniella pulvera & Novgorodova (2005) \\
\hline & Rhopalosiphum padi & Novgorodova (2005) \\
\hline & Schizaphis gramina & Novgorodova (2005) \\
\hline & Schizaphis pyri & Novgorodova (2005) \\
\hline & Symydobius oblongus & Novgorodova (2005) \\
\hline \multirow{8}{*}{ Formica pratensis } & Aphis craccivora & Novgorodova (2005) \\
\hline & Chaitophorus nassonowi & Novgorodova (2005) \\
\hline & Chaitophorus populeti & Novgorodova (2005) \\
\hline & Cinara boerneri & Novgorodova (2005) \\
\hline & Cinara laricis & Novgorodova (2005) \\
\hline & Cinara pinea & Novgorodova (2005) \\
\hline & Cinara pini & Novgorodova (2005) \\
\hline & Symydobius oblongus & Novgorodova (2005) \\
\hline Formica propinqua & Chaitophorus populicola & Wimp and Whitham (2001) \\
\hline
\end{tabular}




\begin{tabular}{|c|c|c|}
\hline \multirow{3}{*}{ Formica puberula } & Aphis salicariae & Addicott (1979a) \\
\hline & Aphis varians & Addicott (1979a) \\
\hline & Rhopalosiphum nymphaeae & Jones (1927) \\
\hline Formica ravida & Rhopalosiphum nymphaeae & Jones (1927) \\
\hline Formica rubicunda & Rhopalosiphum nymphaeae & Jones (1927) \\
\hline \multirow{9}{*}{ Formica rufa } & Aphis helianthi & Mooney et al. (2015) \\
\hline & Aphis medicaginis & Jones (1927) \\
\hline & Aphis salicariae & Mooney et al. (2015) \\
\hline & Aphis valerianae & Jones (1927) \\
\hline & Aphis varians & Mooney et al. (2015) \\
\hline & Brachycaudus cardui & Jones (1927) \\
\hline & Chaitophorus populifolii & Jones (1927) \\
\hline & Nearctaphis bakeri & Jones (1927) \\
\hline & Rhopalosiphum nymphaeae & Jones (1927) \\
\hline \multirow{8}{*}{ Formica rufibarbis } & Aphis pomi & Akyildirim et al. (2014) \\
\hline & Aphis spiraecola & Akyürek et al. (2016) \\
\hline & Cinara indica & Akyürek et al. (2016) \\
\hline & Cinara occidentalis & Akyürek et al. (2016) \\
\hline & Dysaphis devecta & Akyürek et al. (2016) \\
\hline & Myzus ornatus & Akyürek et al. (2016) \\
\hline & Myzus padellus & Akyürek et al. (2016) \\
\hline & Tuberolachnus salignus & Akyürek et al. (2016) \\
\hline \multirow{2}{*}{ Formica sanguinea } & Chaitophorus populeti & Akyürek et al. (2016) \\
\hline & Rhopalosiphum nymphaeae & Jones (1927) \\
\hline Formica subaenescens & Rhopalosiphum nymphaeae & Jones (1927) \\
\hline Formica subintegra & Rhopalosiphum nymphaeae & Jones (1927) \\
\hline \multirow{8}{*}{ Formica subsericea } & Aphis coreopsidis & Favret et al. (2010) \\
\hline & Aulacorthum solani & Favret et al. (2010) \\
\hline & Cinara atlantica & Favret et al. (2010) \\
\hline & Cinara pinivora & Favret et al. (2010) \\
\hline & Hyperomyzus nabali & Favret et al. (2010) \\
\hline & Neomyzus circumflexum & Favret et al. (2010) \\
\hline & Rhopalosiphum nymphaeae & Jones (1927) \\
\hline & Uroleucon pieloui & Favret et al. (2010) \\
\hline Formica ulkei & Rhopalosiphum nymphaeae & Jones (1927) \\
\hline Formica yessensis & Tuberculatus quercicola & $\begin{array}{l}\text { Yao and Akimoto (2001), Yao and Akimoto } \\
\text { (2009) }\end{array}$ \\
\hline \multirow{2}{*}{ Proformica kobachidzei } & Lachnus roboris & Akyildirim et al. (2014) \\
\hline & Myzus cerasi & Akyildirim et al. (2014) \\
\hline Proformica pilosiscapa & Brachycaudus amygdalinus & Mortazavi et al. (2015) \\
\hline \multicolumn{3}{|l|}{ Tribe Lasiini } \\
\hline \multirow{3}{*}{ Lasius alienus } & Aphis fabae & $\begin{array}{c}\text { Akyildirim et al. (2014), Akyürek et al. } \\
\text { (2016) }\end{array}$ \\
\hline & Aphis galliiscabri & Özdemir et al. (2008) \\
\hline & Aphis gossypii & $\begin{array}{c}\text { Akyildirim et al. (2014), Akyürek et al. } \\
\text { (2016) }\end{array}$ \\
\hline
\end{tabular}




\begin{tabular}{|c|c|c|}
\hline & Aphis idaei & Stary (1969) \\
\hline & Aphis maidi-radicis & Forbes (1906) \\
\hline & Aphis molluginis & Akyildirim et al. (2014) \\
\hline & Aphis nasturtii & Özdemir et al. (2008) \\
\hline & Aphis pomi & Akyürek et al. (2016) \\
\hline & Aphis pseudocardui & Akyürek et al. (2016) \\
\hline & Aphis solanella & Akyürek et al. (2016) \\
\hline & Aphis urticata & Akyürek et al. (2016) \\
\hline & Aphis verbasci & Özdemir et al. (2008), Akyürek et al. (2016) \\
\hline & $\begin{array}{c}\text { Brachycaudus (Appelia) } \\
\text { tragopogonis }\end{array}$ & Özdemir et al. (2008) \\
\hline & Brachycaudus cardui & Akyürek et al. (2016) \\
\hline & Capitophorus hippophaes & Özdemir et al. (2008) \\
\hline & Chaitophorus kapuri & Akyildirim et al. (2014) \\
\hline & Cinara atlantica & Favret et al. (2010) \\
\hline & Cinara palaestinesis & LATIBARI et al. (2016) \\
\hline & Cinara pini & LATIBARI et al. (2016) \\
\hline & Dysaphis foeniculus & Akyürek et al. (2016) \\
\hline & Dysaphis pyri & Akyildirim et al. (2014) \\
\hline & Myzus cerasi & Akyürek et al. (2016) \\
\hline & Neobetulaphis pusilla & Akyildirim et al. (2014) \\
\hline & Periphyllus bulgaricus & Mortazavi et al. (2015) \\
\hline & Rhopalosiphum cerasifoliae & Favret et al. (2010) \\
\hline & Rhopalosiphum maidis & Barton and Ives (2014) \\
\hline & Rhopalosiphum nymphaeae & Jones (1927) \\
\hline & Sipha maydis & Akyürek et al. (2016) \\
\hline & Toxoptera aurantii & Akyildirim et al. (2014) \\
\hline \multirow{2}{*}{ Lasius americanus } & Pemphigus lasii & Palmer (1952), Cockerell (1903) \\
\hline & Forda marginata & Cockerell (1903) \\
\hline \multirow{16}{*}{ Lasius brunneus } & Aphis craccivora & Akyürek et al. (2016) \\
\hline & Aphis fabae & Akyürek et al. (2016) \\
\hline & Aphis hillerislambersi & Akyürek et al. (2016) \\
\hline & Aphis middletonii & Akyürek et al. (2016) \\
\hline & Aphis nasturtii & Akyürek et al. (2016) \\
\hline & Aphis pomi & Akyürek et al. (2016) \\
\hline & Aphis spiraecola & Akyürek et al. (2016) \\
\hline & Brachycaudus cardui & Akyürek et al. (2016) \\
\hline & Cinara cedri & Akyürek et al. (2016) \\
\hline & Cinara cupressi & Akyürek et al. (2016) \\
\hline & Cinara tujafilina & Akyürek et al. (2016) \\
\hline & Dysaphis plantaginea & Akyürek et al. (2016) \\
\hline & Hyalopterus pruni & Akyürek et al. (2016) \\
\hline & Lachnus roboris & Akyürek et al. (2016) \\
\hline & Periphyllus aceris & Akyürek et al. (2016) \\
\hline & Pterocomma populeum & Akyürek et al. (2016) \\
\hline
\end{tabular}




\begin{tabular}{|c|c|c|}
\hline & Schizaphis pyri & Akyürek et al. (2016) \\
\hline & Sitobion fragariae & Akyürek et al. (2016) \\
\hline & Thelaxes suberi & Akyürek et al. (2016) \\
\hline & Uroleucon kashmiricum & Akyürek et al. (2016) \\
\hline \multirow{2}{*}{ Lasius claviger } & Forda marginata & Cockerell (1903) \\
\hline & Rhopalosiphum nymphaeae & Jones (1927) \\
\hline \multirow{15}{*}{ Lasius emarginatus } & Anoecia corni & Akyildirim et al. (2014) \\
\hline & Aphis fabae & Akyildirim et al. (2014) \\
\hline & Aphis gossypii & Akyildirim et al. (2014) \\
\hline & Aphis impatientis & Akyildirim et al. (2014) \\
\hline & Aphis longituba & Akyildirim et al. (2014) \\
\hline & Aphis nasturtii & Akyildirim et al. (2014) \\
\hline & Aphis spiraecola & Akyildirim et al. (2014) \\
\hline & Brachycaudus cardui & Akyildirim et al. (2014) \\
\hline & Brachycaudus schwartzi & Akyildirim et al. (2014) \\
\hline & Chaitophorus longisetosus & Akyildirim et al. (2014) \\
\hline & Cinara pini & Akyildirim et al. (2014) \\
\hline & Myzus cerasi & Akyildirim et al. (2014) \\
\hline & Myzus lythri & Akyildirim et al. (2014) \\
\hline & Periphyllus aceris & Akyildirim et al. (2014) \\
\hline & Thelaxes californica & Akyildirim et al. (2014) \\
\hline Lasius flavus & Forda marginata & Cockerell (1903) \\
\hline Lasius fuji & Stomaphis yanonis & Endo and Itino (2013) \\
\hline \multirow{11}{*}{ Lasius fuliginosus } & Chaitophorus populeti & Novgorodova (2005) \\
\hline & Chaitophorus vitellinae & Molnar et al. (2000) \\
\hline & Cinara boerneri & Novgorodova (2005) \\
\hline & Cinara laricis & Novgorodova (2005) \\
\hline & Cinara pinea & Novgorodova (2005) \\
\hline & Lachnus tropicalis & Zhang et al. (2015) \\
\hline & Pterocomma pilosum & Molnar et al. (2000) \\
\hline & Pterocomma rufipes & Molnar et al. (2000) \\
\hline & Stomaphis japonica & Zhang et al. (2015) \\
\hline & Stomaphis quercus & Novgorodova (2005) \\
\hline & Symydobius oblongus & Novgorodova (2005) \\
\hline Lasius grandis & Lachnus roboris & Paris and Espadaler (2009) \\
\hline \multirow{2}{*}{ Lasius japonicus } & Aphis craccivora & Suzuki et al. (2004), Katayama et al. (2013) \\
\hline & Chaitophorus saliniger & Hembry et al. (2006) \\
\hline Lasius latipes & Rhopalosiphum nymphaeae & Jones (1927) \\
\hline \multirow{2}{*}{ Lasius nearcticus } & Rhopalosiphum nymphaeae & Jones (1927) \\
\hline & Lachnus roboris & Paris and Espadaler (2009) \\
\hline Lasius neoniger & Rhopalosiphum nymphaeae & Jones (1927) \\
\hline \multirow{3}{*}{ Lasius niger } & Aphis craccae & Novgorodova (2005) \\
\hline & Aphis craccivora & $\begin{array}{c}\text { Novgorodova (2005), Kataria and Kumar } \\
(2013)\end{array}$ \\
\hline & Aphis fabae & Liepert and Dettner (1993), Volkl et al. \\
\hline
\end{tabular}




\begin{tabular}{|c|c|c|}
\hline & & $\begin{array}{l}\text { (1999), Offenberg (2001), Woodring et al. } \\
\text { (2004), Fischer et al. (2005), Detrain et al. } \\
\text { (2010), Sanders and Frank (2010), Tegelaar } \\
\text { et al. (2012), Tegelaar et al. (2013), Fischer } \\
\text { et al. (2015), Tegelaar (2015), Vantaux et al. } \\
\text { (2015), }\end{array}$ \\
\hline & Aphis gossypii & Kaneko (2003) \\
\hline & Aphis idaei & Novgorodova (2005) \\
\hline & Aphis jacobaeae & $\begin{array}{c}\text { Vrieling et al. (1991), Müller and Godfray } \\
\text { (1999) }\end{array}$ \\
\hline & Aphis pomi & Novgorodova (2005) \\
\hline & Aphis vandergooti & Woodring et al. (2004) \\
\hline & Aphis viburni & Novgorodova (2005) \\
\hline & Brachycaudus cardui & $\begin{array}{l}\text { Volkl et al. (1999), Müller and Godfray } \\
\text { (1999), Woodring et al. (2004) }\end{array}$ \\
\hline & Callipterinella tuberculata & Novgorodova (2005) \\
\hline & Chaitophorus albus & Novgorodova (2005) \\
\hline & Chaitophorus populeti & $\begin{array}{l}\text { Fischer and Shingleton (2001), } \\
\text { Novgorodova (2005) }\end{array}$ \\
\hline & Chaitophorus populialbae & $\begin{array}{c}\text { Fischer and Shingleton (2001), } \\
\text { Novgorodova (2005) }\end{array}$ \\
\hline & Chaitophorus tremulae & Novgorodova (2005) \\
\hline & Chromaphis obiensis & Novgorodova (2005) \\
\hline & Cinara pini & Novgorodova (2005) \\
\hline & Dysaphis plantaginea & Nagy et al. (2015) \\
\hline & Lachnus tropicalis & Sakata (1995) \\
\hline & Metopeurum fuscoviride & $\begin{array}{l}\text { Volkl et al. (1999), Fischer et al. (2002), } \\
\text { Woodring et al. (2004) }\end{array}$ \\
\hline & Myzocallis kuricola & Sakata (1995) \\
\hline & Symydobius oblongus & Novgorodova (2005) \\
\hline & Trama troglodytes & Woodring et al. (2004) \\
\hline Lasius pallitarsis & Rhopalosiphum nymphaeae & Jones (1927) \\
\hline \multirow{15}{*}{ Lasius paralienus } & Aphis craccivora & Özdemir et al. (2008), Shiran et al. (2013) \\
\hline & Aphis fabae & Özdemir et al. (2008), Shiran et al. (2013) \\
\hline & Aphis frangulae & Shiran et al. (2013) \\
\hline & Aphis galliiscabri & Özdemir et al. (2008) \\
\hline & Aphis gossypii & Özdemir et al. (2008) \\
\hline & Aphis rumicis & Özdemir et al. (2008) \\
\hline & Aphis umbrella & Shiran et al. (2013) \\
\hline & Brachycaudus cardui & Özdemir et al. (2008) \\
\hline & Chaitophorus longisetosus & Akyildirim et al. (2014) \\
\hline & Chaitophorus populeti & Shiran et al. (2013) \\
\hline & Chaitophorus populialbae & Shiran et al. (2013) \\
\hline & Chaitophorus remaudierei & Shiran et al. (2013) \\
\hline & Chaitophorus truncates & Shiran et al. (2013) \\
\hline & Cinara palaestinensis & Shiran et al. (2013) \\
\hline & Cinara palaestinesis & LATIBARI et al. (2016) \\
\hline
\end{tabular}




\begin{tabular}{|c|c|c|}
\hline & Cinara pini & LATIBARI et al. (2016) \\
\hline & Hyadaphis foeniculi & Özdemir et al. (2008) \\
\hline & Hyalopterus pruni & Shiran et al. (2013) \\
\hline & Myzus persicae & Shiran et al. (2013) \\
\hline & Pterochloroides persicae & Shiran et al. (2013) \\
\hline Lasius productus & Stomaphis hirukawai & Matsuura and Yashiro (2006) \\
\hline \multirow{30}{*}{ Lasius turcicus } & Acyrthosiphon gossypii & Mortazavi et al. (2015) \\
\hline & Acyrthosiphon pisum & $\begin{array}{c}\text { Akyildirim et al. (2014), Akyürek et al. } \\
\text { (2016) }\end{array}$ \\
\hline & Anoecia corni & Akyildirim et al. (2014) \\
\hline & Aphis brunellae & Akyürek et al. (2016) \\
\hline & Aphis craccivora & $\begin{array}{l}\text { Özdemir et al. (2008), Mortazavi et al. } \\
\text { (2015) }\end{array}$ \\
\hline & Aphis fabae & $\begin{array}{c}\text { Akyildirim et al. (2014), Akyürek et al. } \\
\text { (2016) }\end{array}$ \\
\hline & Aphis farinosa & Akyildirim et al. (2014) \\
\hline & Aphis gossypii & $\begin{array}{l}\text { Akyildirim et al. (2014), Akyürek et al. } \\
\text { (2016) }\end{array}$ \\
\hline & Aphis pomi & Akyürek et al. (2016) \\
\hline & Aphis sambuci & Akyildirim et al. (2014) \\
\hline & Aphis spiraecola & $\begin{array}{c}\text { Akyildirim et al. (2014), Akyürek et al. } \\
\text { (2016) }\end{array}$ \\
\hline & Brachycaudus amygdalinus & Mortazavi et al. (2015) \\
\hline & Brachycaudus cardui & $\begin{array}{c}\text { Akyildirim et al. (2014), Akyürek et al. } \\
\text { (2016) }\end{array}$ \\
\hline & Brachycaudus helichrysi & Akyürek et al. (2016) \\
\hline & Chaetosiphon tetrarhodum & Akyildirim et al. (2014) \\
\hline & $\begin{array}{l}\text { Chaitophorus } \\
\text { hillerislambersi }\end{array}$ & Mortazavi et al. (2015) \\
\hline & Chaitophorus salicti & Akyildirim et al. (2014) \\
\hline & Cinara cupressi & Akyürek et al. (2016) \\
\hline & Cinara pilicornis & Akyildirim et al. (2014) \\
\hline & Cinara pini & Akyildirim et al. (2014) \\
\hline & Cinara tujafilina & Akyürek et al. (2016) \\
\hline & Myzus cerasi & $\begin{array}{c}\text { Akyildirim et al. (2014), Akyürek et al. } \\
\text { (2016) }\end{array}$ \\
\hline & Myzus lythri & Akyildirim et al. (2014) \\
\hline & Myzus varians & Akyürek et al. (2016) \\
\hline & Neobetulaphis pusilla & Akyildirim et al. (2014) \\
\hline & Phleomyzus passerini & Akyildirim et al. (2014) \\
\hline & Protaphis terricola & Özdemir et al. (2008) \\
\hline & Pterochloroides persicae & Akyildirim et al. (2014) \\
\hline & Pterocomma populeum & Akyürek et al. (2016) \\
\hline & Toxoptera aurantii & Akyildirim et al. (2014) \\
\hline \multirow{3}{*}{ Nylanderia fulva } & Aphis craccivora & Sharma et al. (2013) \\
\hline & Aphis vernoniae & Sharma et al. (2013) \\
\hline & Chaitophorus viminicola & Sharma et al. (2013) \\
\hline
\end{tabular}




\begin{tabular}{|c|c|c|}
\hline & Cinara juniperivora & Sharma et al. (2013) \\
\hline & Sanbornia juniperi & Sharma et al. (2013) \\
\hline & Shivaphis celti & Sharma et al. (2013) \\
\hline \multirow{2}{*}{ Paratrechina bourbonica } & Aphis gossypii & Idechiil et al. (2007) \\
\hline & Pentalonia nigronervosa & Idechiil et al. (2007) \\
\hline \multirow{3}{*}{ Paratrechina longicornis } & Aphis coreopsidis & Favret et al. (2010) \\
\hline & Aphis craccivora & $\begin{array}{c}\text { Shiran et al. (2013), Rakhshan and Ahmad } \\
\text { (2015) }\end{array}$ \\
\hline & Aphis gossypii & $\begin{array}{c}\text { Idechiil et al. (2007), Lokeshwari et al. } \\
\text { (2015) }\end{array}$ \\
\hline Paratrechina parvula & Aphis coreopsidis & Favret et al. (2010) \\
\hline Paratrechina vaga & Aphis clerodendri & Carver et al. (2003) \\
\hline \multicolumn{3}{|l|}{ Tribe Melophorini } \\
\hline Notoncus capitatus & Aphis clerodendri & Carver et al. (2003) \\
\hline \multicolumn{3}{|l|}{ Tribe Myrmelachistini } \\
\hline Brachymyrmex patagonica & Brachycaudus cardui & Lescano et al. (2015) \\
\hline \multicolumn{3}{|l|}{ Tribe Plagiolepidini } \\
\hline \multirow{4}{*}{ Acantholepis sp. } & Aphis nerii & Stary (1969) \\
\hline & Chaitophorus sp. & Stary (1969) \\
\hline & Lachnus sp. & Stary (1969) \\
\hline & Thelaxes suberis & Stary (1969) \\
\hline \multirow{2}{*}{ Anoplolepis gracilipes } & Aphis gossypii & Idechiil et al. (2007) \\
\hline & Pentalonia nigronervosa & Idechiil et al. (2007) \\
\hline \multirow{15}{*}{ Lepisiota bipartite } & Aphis craccivora & Shiran et al. (2013) \\
\hline & Aphis fabae & Shiran et al. (2013) \\
\hline & Aphis frangulae & Shiran et al. (2013) \\
\hline & Aphis nasturtii & Shiran et al. (2013) \\
\hline & Aphis nerii & Shiran et al. (2013) \\
\hline & Aphis parietariae & Shiran et al. (2013) \\
\hline & Aphis punicae & Shiran et al. (2013) \\
\hline & Aphis umbrella & Shiran et al. (2013) \\
\hline & Chaitophorus euphraticus & Shiran et al. (2013) \\
\hline & Chaitophorus truncates & Shiran et al. (2013) \\
\hline & Hyalopterus pruni & Shiran et al. (2013) \\
\hline & Myzus persicae & Shiran et al. (2013) \\
\hline & Pterochloroides persicae & Shiran et al. (2013) \\
\hline & Rhopalosiphum maidis & Shiran et al. (2013) \\
\hline & Rhopalosiphum padi & Shiran et al. (2013) \\
\hline \multirow{2}{*}{ Lepisiota dolabellae } & Acyrthosiphon pisum & Mortazavi et al. (2015) \\
\hline & Brachycaudus tragopogonis & Mortazavi et al. (2015) \\
\hline \multirow{2}{*}{ Lepisiota frauenfeldi } & Aphis craccivora & Rakhshan and Ahmad (2015) \\
\hline & Pterochloroides persicae & Mortazavi et al. (2015) \\
\hline \multirow{3}{*}{ Lepisiota nigra } & Aphis fabae & Mortazavi et al. (2015) \\
\hline & Aphis gossypii & Mortazavi et al. (2015) \\
\hline & Brachycaudus helichrysi & Mortazavi et al. (2015) \\
\hline
\end{tabular}




\begin{tabular}{|c|c|c|}
\hline & Brachycaudus tragopogonis & Mortazavi et al. (2015) \\
\hline & Chaitophorus israeliticus & Mortazavi et al. (2015) \\
\hline & Cinara pini & LATIBARI et al. (2016) \\
\hline & Hyalopterus amygdali & Mortazavi et al. (2015) \\
\hline & Macrosiphon rosae & Mortazavi et al. (2015) \\
\hline & Periphyllus bulgaricus & Mortazavi et al. (2015) \\
\hline & Pterochloroides persicae & Mortazavi et al. (2015) \\
\hline & Schizaphis nigerrima & Mortazavi et al. (2015) \\
\hline \multirow{9}{*}{ Plagiolepis pallescens } & Acyrthosiphon pisum & Mortazavi et al. (2015) \\
\hline & Aphis craccivora & Özdemir et al. (2008), Shiran et al. (2013) \\
\hline & Aphis fabae & Shiran et al. (2013) \\
\hline & Aphis frangulae & Shiran et al. (2013) \\
\hline & $\begin{array}{c}\text { Brachycaudus (Appelia) } \\
\text { tragopogonis }\end{array}$ & Özdemir et al. (2008) \\
\hline & Brachycaudus tragopogonis & Mortazavi et al. (2015) \\
\hline & Callaphis juglandis & Mortazavi et al. (2015) \\
\hline & Hyalopterus amygdali & Shiran et al. (2013) \\
\hline & Tinocallis platani & Shiran et al. (2013) \\
\hline \multirow{6}{*}{ Plagiolepis pygmaea } & Aphis craccivora & Mortazavi et al. (2015) \\
\hline & Aphis fabae & Akyürek et al. (2016) \\
\hline & Aphis gerardianae & Akyürek et al. (2016) \\
\hline & Aphis gossypii & Akyürek et al. (2016) \\
\hline & Aphis spiraecola & Akyürek et al. (2016) \\
\hline & Brachycaudus cardui & Akyürek et al. (2016) \\
\hline \multirow{2}{*}{ Plagiolepis taurica } & Aphis spiraecola & Akyildirim et al. (2014) \\
\hline & Toxoptera aurantii & Akyildirim et al. (2014) \\
\hline Plagiolepis vindobonensis & Staegeriella necopinata & Özdemir et al. (2008) \\
\hline \multicolumn{3}{|l|}{ Subfamily Myrmicinae } \\
\hline \multicolumn{3}{|l|}{ Tribe Attini } \\
\hline \multirow{2}{*}{ Pheidole bicarinata } & Aphis asclepiadis & Jones (1927) \\
\hline & Thecabius populimonilis & Jones (1927) \\
\hline Pheidole fervens & Aphis gossypii & Idechiil et al. (2007) \\
\hline \multirow{7}{*}{ Pheidole pallidula } & Aphis davletshinae & Shiran et al. (2013) \\
\hline & Aphis frangulae & Shiran et al. (2013) \\
\hline & Aphis umbrella & Shiran et al. (2013) \\
\hline & Cinara palaestinesis & LATIBARI et al. (2016) \\
\hline & Myzus persicae & Shiran et al. (2013) \\
\hline & Aphis nerii & Stary (1969) \\
\hline & Thelaxes suberis & Stary (1969) \\
\hline Pheidole pilifera & $\begin{array}{c}\text { Pemphigus } \\
\text { populiramulorum }\end{array}$ & Jones (1927) \\
\hline \multirow{2}{*}{ Pheidole teneriffana } & Aphis craccivora & Shiran et al. (2013) \\
\hline & Aphis umbrella & Shiran et al. (2013) \\
\hline \multicolumn{3}{|l|}{ Tribe Crematogastrini } \\
\hline Cardiocondyla emeryi & Pentalonia nigronervosa & Idechiil et al. (2007) \\
\hline
\end{tabular}




\begin{tabular}{|c|c|c|}
\hline Cardiocondyla shalbergi & Cinara pini & LATIBARI et al. (2016) \\
\hline Cardiocondyla wroughtonii & Pentalonia nigronervosa & Idechiil et al. (2007) \\
\hline \multirow{4}{*}{ Cremastogaster scutellaris } & Aphis fabae & Akyürek et al. (2016) \\
\hline & Aphis pomi & Akyürek et al. (2016) \\
\hline & Aphis spiraecola & Akyürek et al. (2016) \\
\hline & Pterocomma rufipes & Akyürek et al. (2016) \\
\hline \multirow{5}{*}{ Crematogaster antaris } & Aphis craccivora & Shiran et al. (2013) \\
\hline & Aphis farinosa & Shiran et al. (2013) \\
\hline & Aphis frangulae & Shiran et al. (2013) \\
\hline & Chaitophorus truncates & Shiran et al. (2013) \\
\hline & Myzus persicae & Shiran et al. (2013) \\
\hline Crematogaster auberti & Thelaxes suberis & Stary (1969) \\
\hline \multirow{8}{*}{ Crematogaster inermis } & Acyrthosiphon gossypii & Mortazavi et al. (2015) \\
\hline & Acyrthosiphon pisum & Mortazavi et al. (2015) \\
\hline & Aphis craccivora & Mortazavi et al. (2015) \\
\hline & Aphis gossypii & Mortazavi et al. (2015) \\
\hline & Aphis pseudocardui & Mortazavi et al. (2015) \\
\hline & Brachycaudus tragopogonis & Mortazavi et al. (2015) \\
\hline & Callaphis juglandis & Mortazavi et al. (2015) \\
\hline & Chaitophorus israeliticus & Mortazavi et al. (2015) \\
\hline Crematogaster laeviuscula & Cinara ponderosae & Jones (1927) \\
\hline \multirow{9}{*}{ Crematogaster lineolata } & Aphis coreopsidis & Favret et al. (2010) \\
\hline & Aphis fabae & Favret et al. (2010) \\
\hline & Aphis gossypii & Favret et al. (2010) \\
\hline & Capitophorus elaeagni & Favret et al. (2010) \\
\hline & Chaitophorus viminicola & Favret et al. (2010) \\
\hline & Cinara edulis & Jones (1927) \\
\hline & Cinara murrayanae & Jones (1927) \\
\hline & Cinara schwarzii & Jones (1927) \\
\hline & Lachnus ater & Jones (1927) \\
\hline \multirow{4}{*}{ Crematogaster schmidti } & Aphis craccivora & Mortazavi et al. (2015) \\
\hline & Aphis pseudocardui & Mortazavi et al. (2015) \\
\hline & Chaitophorus israeliticus & Mortazavi et al. (2015) \\
\hline & Thelaxes californica & Akyildirim et al. (2014) \\
\hline Crematogaster scutellaris & Chaitophorus melanosiphon & Akyürek et al. (2016) \\
\hline \multirow[t]{2}{*}{ Crematogaster sordidula } & Brachycaudus cardui & $\begin{array}{c}\text { Özdemir et al. (2008), Mortazavi et al. } \\
\text { (2015) }\end{array}$ \\
\hline & Thelaxes suberis & Stary (1969) \\
\hline \multirow{4}{*}{ Crematogaster subdentata } & Aphis craccivora & Mortazavi et al. (2015) \\
\hline & Cinara pini & LATIBARI et al. (2016) \\
\hline & Periphyllus bulgaricus & Mortazavi et al. (2015) \\
\hline & Tuberculatus maximus & Mortazavi et al. (2015) \\
\hline Crematogaster warburgi & Chaitophorus sp. & Stary (1969) \\
\hline Leptothorax muscorum & Rhopalosiphum nymphaeae & Jones (1927) \\
\hline Lophomyrmex & Aphis gossypii & Lokeshwari et al. (2015) \\
\hline
\end{tabular}




\begin{tabular}{|c|c|c|}
\hline quadrispinosus & & \\
\hline Meranoplus bicolor & Aphis craccivora & Rakhshan and Ahmad (2015) \\
\hline \multirow{2}{*}{ Myrmecina graminicola } & Aphis forbesi & Jones (1927) \\
\hline & Chaitophorus viminalis & Jones (1927) \\
\hline Pristomyrmex punctatus & Aphis craccivora & Katayama et al. (2013) \\
\hline Pristomyrmex pungens & Aphis gossypii & Kaneko (2003) \\
\hline \multirow{2}{*}{ Tetramorium bicarinatum } & Aphis gossypii & Idechiil et al. (2007) \\
\hline & Pentalonia nigronervosa & Idechiil et al. (2007) \\
\hline \multirow{8}{*}{ Tetramorium caespitum } & Aphis fabae & Shiran et al. (2013), Akyildirim et al. (2014) \\
\hline & Aphis farinosa & Akyildirim et al. (2014) \\
\hline & Aphis gossypii & Akyildirim et al. (2014) \\
\hline & Aphis salviae & Akyürek et al. (2016) \\
\hline & Aphis spiraecola & Akyürek et al. (2016) \\
\hline & Brachycaudus cardui & Akyürek et al. (2016) \\
\hline & Macrosiphoniella sanborni & Akyildirim et al. (2014) \\
\hline & Myzus lythri & Akyürek et al. (2016) \\
\hline \multirow[t]{2}{*}{ Tetramorium chefteki } & Aphis craccivora & $\begin{array}{c}\text { Özdemir et al. (2008), Mortazavi et al. } \\
\text { (2015) }\end{array}$ \\
\hline & Aphis rumicis & Özdemir et al. (2008) \\
\hline \multirow{5}{*}{ Tetramorium forte } & Aphis craccivora & Özdemir et al. (2008) \\
\hline & Aphis rumicis & Özdemir et al. (2008) \\
\hline & Brachycaudus cardui & Akyildirim et al. (2014) \\
\hline & Cinara pilicornis & Akyildirim et al. (2014) \\
\hline & Uroleucon sonchi & Akyürek et al. (2016) \\
\hline Tetramorium semilaeve & Paracletus cimiciformis & Salazar et al. (2015) \\
\hline \multirow[t]{2}{*}{ Tetramorium tsushimae } & Aphis craccivora & $\begin{array}{l}\text { Suzuki et al. (2004), Katayama et al. (2013), } \\
\text { Hayashi et al. (2015), Hayashi et al. (2016) }\end{array}$ \\
\hline & Aphis fabae & Hayashi et al. (2015) \\
\hline \multicolumn{3}{|l|}{ Tribe Myrmicini } \\
\hline \multirow{16}{*}{ Myrmica incompleta } & Aphis asclepiadis & Jones (1927) \\
\hline & Aphis gossypii & Jones (1927) \\
\hline & Aphis medicaginis & Jones (1927) \\
\hline & Aphis pomi & Jones (1927) \\
\hline & Aphis populicola & Jones (1927) \\
\hline & Aphis rumicis & Jones (1927) \\
\hline & Artemisaphis artemisicola & Jones (1927) \\
\hline & Brachycaudus cardui & Jones (1927) \\
\hline & Brachycaudus prunicola & Jones (1927) \\
\hline & $\begin{array}{l}\text { Chaitophorus } \\
\text { macrostachyae }\end{array}$ & Jones (1927) \\
\hline & Chaitophorus populicola & Jones (1927) \\
\hline & Cinara oregonensis & Jones (1927) \\
\hline & Eriosoma crataegi & Jones (1927) \\
\hline & Protaphis middletonii & Jones (1927) \\
\hline & Pterocomma bicolor & Jones (1927) \\
\hline & Pterocomma salicis & Jones (1927) \\
\hline
\end{tabular}




\begin{tabular}{|c|c|c|}
\hline & Rhopalosiphum maidis & Jones (1927) \\
\hline & Uroleucon ambrosiae & Jones (1927) \\
\hline & Wahlgreniella nervata & Jones (1927) \\
\hline \multirow{3}{*}{ Myrmica punctiventris } & Aphis illinoisensis & Favret et al. (2010) \\
\hline & Aphis lugentis & Favret et al. (2010) \\
\hline & Drepanaphis nigricans & Favret et al. (2010) \\
\hline Myrmica rubra & Cinara apini & Jones (1927) \\
\hline \multirow{3}{*}{ Myrmica ruginodis } & Aphis fabae & Akyildirim et al. (2014) \\
\hline & Aphis jacobaeae & Müller and Godfray (1999) \\
\hline & Brachycaudus cardui & $\begin{array}{c}\text { Müller and Godfray (1999), Akyildirim et al. } \\
\text { (2014) }\end{array}$ \\
\hline \multirow{7}{*}{ Myrmica scabrinodis } & Aphis asclepiadis & Jones (1927) \\
\hline & Aphis forbesi & Jones (1927) \\
\hline & Brachycaudus helichrysi & Akyildirim et al. (2014) \\
\hline & Brachycaudus persicae & Jones (1927) \\
\hline & Cinara brevispinosa & Jones (1927) \\
\hline & Cryptomyzus ribis & Jones (1927) \\
\hline & Drepanaphis acerifoliae & Jones (1927) \\
\hline \multirow{5}{*}{ Myrmica whymperi } & Aphis asclepiadis & Jones (1927) \\
\hline & Aphis farinosa & Jones (1927) \\
\hline & Aphis oenotherae & Jones (1927) \\
\hline & Aphis rumicis & Jones (1927) \\
\hline & Chaitophorus viminalis & Jones (1927) \\
\hline \multirow{2}{*}{ Pogonomyrmex occidentalis } & Hyalopterus pruni & Jones (1927) \\
\hline & Myzus persicae & Jones (1927) \\
\hline \multicolumn{3}{|l|}{ Tribe Solenopsidini } \\
\hline \multirow{3}{*}{ Monomorium destructor } & Aphis craccivora & Shiran et al. (2013) \\
\hline & Aphis frangulae & Shiran et al. (2013) \\
\hline & Chaitophorus euphraticus & Shiran et al. (2013) \\
\hline Monomorium floricola & Aphis gossypii & Idechiil et al. (2007) \\
\hline \multirow{2}{*}{ Monomorium gracillimum } & Aphis nerii & Stary (1969) \\
\hline & Uroleucon Dactynotus & Stary (1969) \\
\hline Monomorium latinode & Aphis craccivora & Rakhshan and Ahmad (2015) \\
\hline \multirow{2}{*}{ Monomorium libanicum } & Aphis fabae & Shiran et al. (2013) \\
\hline & Aphis frangulae & Shiran et al. (2013) \\
\hline \multirow{9}{*}{ Monomorium mayri } & Aphis craccivora & Shiran et al. (2013) \\
\hline & Aphis fabae & Shiran et al. (2013) \\
\hline & Aphis frangulae & Shiran et al. (2013) \\
\hline & Aphis nerii & Shiran et al. (2013) \\
\hline & Aphis polygonata & Shiran et al. (2013) \\
\hline & Chaitophorus euphraticus & Shiran et al. (2013) \\
\hline & Hyalopterus amygdali & Shiran et al. (2013) \\
\hline & Myzus persicae & Shiran et al. (2013) \\
\hline & Rhopalosiphum maidis & Shiran et al. (2013) \\
\hline Monomorium minimum & Aphis craccivora & Kataria and Kumar (2013) \\
\hline
\end{tabular}




\begin{tabular}{|c|c|c|}
\hline & Aphis fabae & Kataria and Kumar (2013) \\
\hline & Aphis gossypii & Kataria and Kumar (2013) \\
\hline & Aphis nerii & Kataria and Kumar (2013) \\
\hline & Rhopalosiphum nymphaeae & Jones (1927) \\
\hline Monomorium monomorium & Aphis gossypii & Idechiil et al. (2007) \\
\hline Monomorium nitidiventre & Aphis craccivora & Mortazavi et al. (2015) \\
\hline Monomorium pharaonis & Aphis craccivora & Rakhshan and Ahmad (2015) \\
\hline \multirow{3}{*}{ Monomorium qarahe } & Aphis fabae & Shiran et al. (2013) \\
\hline & Aphis frangulae & Shiran et al. (2013) \\
\hline & Rhopalosiphum maidis & Shiran et al. (2013) \\
\hline Solenopsis geminata & Aphis gossypii & $\begin{array}{c}\text { Idechiil et al. (2007), Lokeshwari et al. } \\
\text { (2015) }\end{array}$ \\
\hline Solenopsis invicta & Myzus persicae & Zhou (2012), Zhou (2014) \\
\hline Solenopsis richteri & Brachycaudus cardui & Lescano et al. (2015) \\
\hline Messor orientalis & Chaitophorus israeliticus & Mortazavi et al. (2015) \\
\hline Myrmicaria brunnea & Aphis gossypii & Lokeshwari et al. (2015) \\
\hline \multicolumn{3}{|l|}{ Tribe Stenammini } \\
\hline Aphaenogaster kurdica & Brachycaudus helichrysi & Mortazavi et al. (2015) \\
\hline \multicolumn{3}{|l|}{$\begin{array}{c}\text { Subfamily } \\
\text { Pseudomyrmecinae }\end{array}$} \\
\hline Tetraponera rufonigra & Aphis craccivora & Rakhshan and Ahmad (2015) \\
\hline
\end{tabular}

